This is a post-peer-review, pre-copyedit version of an article published in "Journal of Philosophical Logic". The final authenticated version is available online at: https://doi.org/10.1007/s10992-016-9410-1

This version is subjected to Springer Nature terms for reuse that can be found at: https://www.springer.com/gp/openaccess/authors-rights/aam-terms-v1 


\title{
Quantification in Some Non-normal Modal Logics
}

\author{
Erica Calardo and Antonino Rotolo* \\ CIRSFID - University of Bologna, Italy \\ erica.calardo@unibo.it, antonino.rotolo@unibo.it
}

August 5, 2016

Keywords: Quantified non-normal modal logics, Multi-relational semantics, Completeness, Barcan schemata

\begin{abstract}
This paper offers a semantic study in multi-relational semantics of quantified N-Monotonic modal logics with varying domains with and without the identity symbol. We identify conditions on frames to characterise Barcan and Ghilardi schemata and present some related completeness results. The characterisation of Barcan schemata in multi-relational frames with varying domains shows the independence of $\mathbf{B F}$ and $\mathbf{C B F}$ from well-known propositional modal schemata, an independence that does not hold with constant domains. This fact was firstly suggested for classical modal systems by Stolpe (2003), but unfortunately that work used only models and not frames.
\end{abstract}

\section{Introduction}

A number of significant contributions in the last four decades show that non-normal modal logics (NMLs) can be fruitfully employed in several fields. One well-known domain is epistemic logic, where NMLs are a solution to alleviate the so-called omniscience problem that affects stronger (normal) modal systems (Fagin et al., 1995). Deontic logic is another field where NMLs have been traditionally proposed to avoid many drawbacks of standard deontic logic (i.e., deontic KD), which does not tolerate deontic conflicts and gives rise to a number of paradoxes (Goble, 2005 Jones and Carmo, 2002). Other important applications are those systems capturing different aspects of the concepts of action and agency: The modal logic of agency (Elgesem, 1997, Governatori and Rotolo, 2005, Segerberg, 1992), concurrent propositional dynamic logic (Goldblatt,|1992), game logic (Parikh| 1985), and coalition logic (Pauly, 2002), among others, are all examples where some modal operators are axiomatized in logics weaker than $\mathbf{K}$.

Quantified modal logic has also a long and distinguished tradition (Garson, 2001, Fitting and Mendelsohn, 1998), which is still lively and technically productive (see, among others, Corsi | 2002, Brauner and Ghilardi. 2007, Gabbay et al. 2009 Goldblatt, 2011). Nevertheless, almost all efforts have so far been devoted to the analysis of the normal case: Besides a few significant exceptions (Arló-Costa and Pacuit, 2006 Arló-Costa, 2002, Stolpe, 2003. Waagbø, 1992), which are based on neighbourhood semantics, the study of quantification

*Partially supported the UNIBO project FARB 2012 Mortality Salience, Legal and Social Compliance, and Economic Behaviour: Theoretical Models and Experimental Methods and by the EU H2020 research and innovation programme under the Marie Sklodowska-Curie grant agreement No. 690974 for the project MIREL: MIning and REasoning with Legal texts. 
in non-normal modal logics is still neglected. Despite that, quantified non-normal modal logics (QNML henceforth) exhibit a different behaviour with respect to normal modal logics: For instance the Barcan and the Converse Barcan schemata (i) are not characterised by decreasing and increasing domains (ii) are tightly connected to the validity of propositional modal axiom schemata.

This paper provides a semantic analysis of quantification in the system MN, i.e., a class of non-normal modal logics called N-Monotonic (Calardo and Rotolo, 2014 Chellas, 1980). This class is characterised by the inference rules $\mathbf{R M}$ and $\mathbf{R N}$ or, equivalently, by adding to $\mathrm{E}$ (i.e., the minimal classical modal logic closed under logical equivalence) the axiom schemata $\mathbf{M}$ and $\mathbf{N}$.

$$
\begin{array}{ll}
\mathbf{R M}:=\vdash A \rightarrow B \Rightarrow \vdash \square A \rightarrow \square B & \mathbf{R E}:=\vdash A \leftrightarrow B \Rightarrow \vdash \square A \leftrightarrow \square B \\
\mathbf{R N}:=\vdash A \Rightarrow \vdash \square A & \mathbf{M}:=\square(A \wedge B) \rightarrow(\square A \wedge \square B) \\
& \mathbf{N}:=\square \top
\end{array}
$$

However, instead of working in the standard setting of neighborhood semantics (Chellas 1980 Hansen, 2003 Montague, 1970 Scott, 1970 Segerberg, 1971), we shall focus on multirelational semantics. From the structural point of view, this setting looks like a direct generalization of mono-relational (Kripke) semantics for normal modal logics. A multirelational model is a structure $\langle W, \mathcal{R}, V\rangle$ where $W$ is a set of possible worlds, $\mathcal{R}$ is a (possibly infinite) set of binary relations on $W$, and $V$ is the usual evaluation function of Kripke semantics. Starting from the works of Jennings and Schotch (1981a b) and Goble (2001. 2003, 2004a), two different versions of it have been later identified in the literature (cf. Calardo and Rotolo, 2014, Governatori and Rotolo, 2005), depending on how to evaluate $\square$-formulae.

Here we shall only present a weak formulation of multi-relational semantics, namely the one that simply extends the case of Kripke semantics (Goble, 2001, 2004b, Jennings and Schotch, 1981a b), since it requires that the Kripke-style evaluation clause for $\square$-formulae is satisfied for at at least one relation in the set of relations of the model. In other words:

$$
w \vDash \square \phi \text { iff } \exists R_{j} \in \mathcal{R}: \forall w^{\prime}\left(\text { if } w R_{j} w^{\prime} \text { then } w^{\prime} \vDash \phi\right)
$$

It is plain that this evaluation clause directly generalizes the one for Kripke semantics ${ }^{2}$ since it simply requires that the Kripke-style evaluation clause is satisfied for at least one relation in the set of relations in the model ${ }^{3}$

The aim of this paper is to study quantification within this semantic setting. We consider frames with varying domains and formal languages with and without identity. Thus, we address research issues not yet explored in the literature, while keeping the intuition behind Kripke semantics although working with logics that are stronger than classical systems (E and above), yet strictly weaker than K. On the other hand, working with varying domains (i.e., with sets of individual existing in possible worlds that can vary from world to world) technically amounts to studying the most general case of quantified modal logics, which is

\footnotetext{
${ }^{1}$ In Section 3 the reader can find two tables and a figure classifying the main systems and schemata.

${ }^{2}$ For this reason, throughout the paper we will use interchangeably the expressions "Kripke frame/model", "relational frame/model", and "1-relational frame/model".

${ }^{3}$ The strong version, on the other hand, is as follows (Governatori and Rotolo 2005):

$$
w \vDash \square \phi \text { iff } \exists R_{j} \in \mathcal{R}: \forall w^{\prime}\left(w R_{j} w^{\prime} \text { iff } w^{\prime} \vDash \phi\right)
$$

Things here are the same as in (1), except that $\phi$ is required to be true in $w^{\prime}$ if and only if $w^{\prime}$ is related with $w$ by any relation $R_{j}$. The technical motivation of this second choice is that the resulting semantics is appropriate for any (non-normal) modal logics including the classical ones, i.e., the weakest ones that only consist of $\mathbf{R E}, \vdash A \equiv B / \vdash \square A \equiv \square B$. On the contrary, the version with (1) validates, among others, RM, i.e., $\vdash A \rightarrow B / \vdash \square A \rightarrow \square B$, which opens the door to stronger logics below system $\mathbf{K}$. However, multi-relational models based on the clause 21) make the semantics different from Kripke's, despite the structural similarity of how worlds are connected in frames.
} 
mostly neglected in the literature on modal predicate logics weaker than K. Finally, the role of the identity symbol in QNML is still entirely overlooked: Indeed, identity plays a central technical role in ensuring completeness results.

Paper outline The layout of the paper is as follows.

Section 2 introduces the state of the art and some results concerning quantified non-normal modal logics and neighborhood frames, as well as a first technical introduction to Barcan formulae and the problems related to such schemata.

Section 3 provides some preliminary material and useful auxiliary results from (Calardo and Rotolo, 2014);

Section 4 presents multi-relational first-order frames. We choose to analyse frames with varying domains, which correspond to the most general case and allow for offering a finer distinction between actual individuals and possibilia, namely, between the individuals that exist in each possible world and those that are only possible from that viewpoint, though not actually existing there (Garson, 2001; Fitting and Mendelsohn 1998). Also, this choice technically amounts to studying the most general case of quantified modal logics.

Section 5 analyses alternative semantic characterisations for the Converse Barcan schema (CBF). After comparing our results to the standard ones in Kripke semantics, we shall see different ways to generalise the concept of increasing inner domains.

Section 6 provides Henkin-style completeness theorems for several systems, namely, the smallest free quantified non-normal $\mathrm{N}$-monotonic logic $\mathrm{Q}_{=}^{\circ} . \mathrm{NM}$ and some extensions, including $\mathrm{Q}_{=}^{\circ} . \mathrm{NM} \oplus \mathbf{C B F}$.

Section 7 offers a critical analysis and technical considerations on the Barcan schema BF. This schema raises some interesting questions in the context of non-normal modal logics and in multi-relational semantics.

Section 8 discusses the technical role of the identity relation. As we shall argue, the choice of a language with identity does not only increase the expressive power of the logic, but facilitates the construction of canonical models for CBF-frames, a potentially difficult goal to be achieved without it.

To the best of our knowledge, this is the first study on quantification in multi-relational semantics, the second one investigating the case of varying domains in non-normal modal logics, and the first that provides a frame characterization of Barcan schemata with varying domains with a language with identity.

\section{Neighbourhood Semantics for QNML}

Neighbourhood semantics - and the equivalent strong version of multi-relational semantics (cf. Governatori and Rotolo, 2005) - is in our opinion the best tool to deal with classical systems and above. For this reason, it has always played a central role within non-normal logics. Below we shall summarise the most influential results within this area. This approach will be useful to carry out a comparison between neighbourhood structures and multi-relational ones.

\subsection{Syntax of Quantified Modal Logics}

Let us extend the standard language $\mathcal{L}$ of propositional modal logic (Blackburn et al. 2001) with the universal quantifier $\forall$, a countable set of individual constants Const: $=\{a, b, c, \ldots\}$, a set of individual variables, VAR: $=\{x, y, z, \ldots\}$, the identity predicate $=$, and a set of $n$-ary predicate symbols (where $\omega>n \geq 1$ ). A term is either a variable, or an individual constant and $t_{1}, t_{2}, \ldots$ are meta-variables for terms.

Well formed formuale (wff) are defined as usual: 
(a) $\perp$ is wff;

(b) If $P^{n}$ is an $n$-ary predicate symbol and $t_{1}, \ldots, t_{n}$ are terms, then $P^{n}\left(t_{1}, \ldots, t_{n}\right)$ is a wff;

(c) If $A$ and $B$ are wff, then $A \rightarrow B, \square A$, and $\forall x A$ are wff;

(d) Nothing else is a wff.

Both boolean operators, and the existential quantifier $\exists$ are defined as usual: $\exists x A \leftrightarrow$ $\neg \forall x \neg A$. Moreover $A(t / s)$ is the formula obtained by replacing in $A(s)$ all the free occurrences of $s$ with $t$ (cf. Corsi, 2002, 1484).

\subsection{Neighbourhood Models}

Neighbourhood semantics for quantified modal logics was introduced a long time ago (see Gabbay, 1976). Nevertheless, it received very little attention until the beginning of the 1990s (cf. Waagbø, 1992). The study of QNML became then the subject of a few works (cf., for instance, Arló-Costa, 2002, Arló-Costa and Pacuit, 2006, Stolpe, 2003). While Waagb $\varnothing$ (1992), Arló-Costa (2002), and Arló-Costa and Pacuit (2006) study the case of structures with constant domains, Stolpe (2003) developed a preliminary investigation of varying domains. In this section we summarise their main results.

Let us consider the case of constant domains.

Definition 2.1 (Constant domain neighbourhood frames and models) $A$ constant domain neighbourhood frame $\mathcal{F}$ is a structure $\langle W, \mathcal{N}, D\rangle$ where

- W is a non-empty set of possible worlds;

- $\mathcal{N}$ is a function from $w$ to $2^{2^{W}}$;

- $D$ is a non-empty set of individuals (the domain of the frame).

For any $w \in W$, a $w$-assignment $\sigma$ is a function $\sigma: \operatorname{Var}(\mathcal{L}) \mapsto D$

An $x$-variant $\tau$ of a $w$-assignment $\sigma$ is a $w$-assignment which may differ from $\sigma$ for the value assigned to $x$.

$A$ constant domain neighbourhood model $\mathcal{M}$ is a structure $\langle W, \mathcal{N}, D, I\rangle$ where $\langle W, \mathcal{N}, D\rangle$ is a constant domain neighbourhood frame and $I$ is an interpretation function such that, for any assignment $\sigma$ and world $w$ :

- $I_{w}^{\sigma}(x) \in D$ (global interpretation of variables/terms);

- $\forall w, v \in W, I_{w}^{\sigma}(x)=I_{v}^{\sigma}(x)$ (rigidity of variables/terms);

- $I_{w}^{\sigma}\left(P\left(x_{1}, \ldots, x_{n}\right)\right) \subseteq D^{n}$.

Notice that the notion of truth set has to take into account that the truth or falsity of open formulae depends on particular interpretations.

Definition 2.2 Let $\mathcal{M}$ be a model with interpretation $I, \sigma$ an assignment, $w$ any world, and $A$ any formula. The truth set of $A$ with respect to to $\mathcal{M}$ and $I^{\sigma},\|A\|_{I}^{\sigma}$ is thus defined $\|^{4}$

$$
\|A\|_{I}^{\sigma}:=\left\{w \in W: \mathcal{M} \vDash_{w}^{\sigma} A\right\} .
$$

The valuation conditions are as follows:

- $\mathcal{M} \vDash_{w}^{\sigma} P\left(x_{1}, \ldots, x_{n}\right)$ iff $\left\langle I_{w}^{\sigma}\left(x_{1}\right), \ldots, I_{w}^{\sigma}\left(x_{n}\right)\right\rangle \in I_{w}(P)$;

- Standard valuation conditions for negation and boolean connectives;

- $\mathcal{M} \vDash_{w}^{\sigma} \square A$ iff $\|A\|_{I}^{\sigma} \in \mathcal{N}(w)$;

- $\mathcal{M} \vDash_{w}^{\sigma} \forall x A(x)$ iff, for every $x$-variant $\tau$ of $\sigma, \mathcal{M} \vDash_{w}^{\tau} A(x)$.

Let us consider the Barcan and the Converse Barcan schemata

\footnotetext{
${ }^{4}$ When clear form the context, we also omit the reference to the model. The truth set of a closed formula does not depend on any interpretation and assignment.
} 
The choice of constant domains (i.e., that the resulting modal logics are extensions of standard First Order Logic, FOL henceforth), does not correspond to the validity of BF and CBF:

Theorem 2.3 Arló-Costa and Pacuit, 2006) The class of all constant domain neighbourhood frames is sound and complete for $\mathrm{FOL} \oplus \mathrm{E}$.

BF and CBF were characterised in (Waagbø, 1992), but such results have been later made more precise in (Arló-Costa, 2002, Arló-Costa and Pacuit, 2006). 2006):

Let us consider the following frame properties (Arló-Costa, 2002, Arló-Costa and Pacuit

Definition 2.4 (Frame properties) A frame is consistent iff $\forall w \in W: \mathcal{N}(w) \neq \varnothing$ and $\{\varnothing\} \notin \mathcal{N}(w)$.

A frame is closed under $\leq \kappa$ intersections (where $\kappa$ is a cardinal) iff

$$
\forall w \in W, \forall X=\left\{\mathcal{X}_{i} \mid i \in I\right\} \text { where }|I| \leq \kappa, \bigcap_{i \in I} \mathcal{X}_{i} \in \mathcal{N}(w) .
$$

A frame is trivial iff $|D|=1$, otherwise it is non-trivial.

A frame is supplemented iff $\forall w \in W, X \cap Y \in \mathcal{N}(w) \Rightarrow X \in \mathcal{N}(w)$ and $Y \in \mathcal{N}(w)$.

Theorem 2.5 (Arló-Costa (2002)) BF is valid in the class of frames that are either (i) trivial, or (ii) closed under finite intersection, if $D$ is finite, or (iii) closed under $\leq \kappa$ intersections, if $D$ is infinite and $|D|=\kappa$.

Theorem 2.6 (Waagb $\varnothing$ (1992); Arló-Costa and Pacuit (2006)) CBF is valid in the class of frames that are either supplemented or trivial.

Provided that frames are not trivial, Theorem 2.6 establishes a strong relationship between $\mathbf{M}$ and $\mathbf{C B F}$, since supplementation characterises $\mathbf{M}$. Hence, for constant non-trivial domain neighbourhood frames $\mathbf{C B F}$ is valid whenever $\mathbf{M}$ is. Also, under the same nontriviality assumption, since the closure under $\leq \kappa$ intersections implies the closure under intersection, it is not possible to falsify $\mathbf{C}$ when $\mathbf{B F}$ is valid. However, from the above theorem we can build a countermodel for BF given $\mathbf{C}$ (Waagb $\varnothing, 1992)$, although this is possible only for infinite frames (Arló-Costa, 2002).

An open problem of this semantics is that the system $\mathrm{FOL} \oplus \mathrm{E} \oplus \mathbf{C B F}$ is strongly complete with respect to the class of frames that are either trivial, or supplemented: Arló-Costa (2011) conjectured that $\mathbf{M}$ is thus derivable by adding in the logic a schema expressing non-triviality but no result is available.

Let us now move to the case of varying domains, which was explored in QNML only by Stolpe (2003). The peculiarity of Stolpe (2003)'s analysis is that it works only with models and not with frames. Models are standardly defined as follows:

Definition 2.7 (Varying domain neighbourhood models) $A$ varying domain neighbourhood frame $\mathcal{M}$ is a structure $\langle W, \mathcal{N}, D, \Sigma, I\rangle$ where

- $W, \mathcal{N}, D$, and I are like in Definition 2.1 and

- $\Sigma$ is a function assigning to each world $w \in W$ a set $D_{w}$ of elements of $D$.

The valuation condition for $\forall$-formulae is now as follows:

$\mathcal{M} \vDash_{w}^{\sigma} \forall x A(x)$ iff $\mathcal{M} \vDash_{w}^{\tau} A(x)$ for every $x$-variant $\tau$ of $\sigma$ such that $\tau(x) \in D_{w}$.

Stolpe (2003) defines two classes of varying neighbourhood models that characterize BF and $\mathbf{C B F}$ : 
Theorem 2.8 (BF and CUPI models) $A$ varying neighbourhood model $\mathcal{M}=$ $\langle W, \mathcal{N}, D, I, \Sigma\rangle$ is a CUPI model iff for any world $w \in W$, if $\|P(x)\|_{I}^{\sigma} \in \mathcal{N}(w)$ for every $\sigma$ such that $\sigma(x) \in D_{w}$, then $\|\forall x P(x)\|_{I} \in \mathcal{N}(w)$.

$\mathbf{B F}$ is valid in the class of CUPI models.

Theorem 2.9 (CBF and CUPO models) A varying neighbourhood model $\mathcal{M}=$ $\langle W, \mathcal{N}, D, I, \Sigma\rangle$ is a CUPO model iff for any world $w \in W$, if $\|\forall x P(x)\|_{I} \in \mathcal{N}(w)$, then $\|P(x)\|_{I}^{\sigma} \in \mathcal{N}(w)$ for every $\sigma$ such that $\sigma(x) \in D_{w}$.

$\mathbf{C B F}$ is valid in the class of CUPO models.

CUPI and CUPO models impose properties that trivially reflect the evaluation of BF and CBF. The main limit of this approach is that it does not appeal to frames. Thus, ArlóCosta (2011) rightly argues that Stolpe (2003) leaves open many questions, including the general characterization of $\mathbf{B F}$ and $\mathbf{C B F}$. In this paper, we address this very problem in quantified $\mathbf{N}$-Monotonic logics with varying domains.

\section{Preliminaries: Multi-relational Semantics for the Proposi- tional Case}

In this section, we present some basic notions and results by Calardo and Rotolo (2014) for non-normal propositional modal logics and multi-relational semantics. The alphabet of the language includes as usual a countable set of propositional letters, modal and Boolean operators.

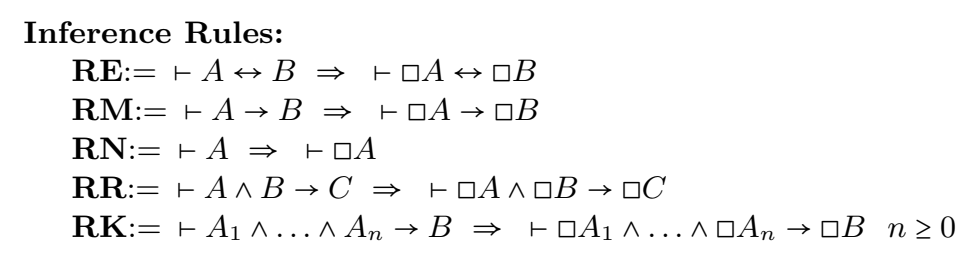

\section{Axiom Schemata:}

$$
\begin{aligned}
& \text { EFQ: }=A \wedge \neg A \rightarrow B \\
& \mathbf{M}:=\square(A \wedge B) \rightarrow(\square A \wedge \square B) \\
& \mathbf{C}:=(\square A \wedge \square B) \rightarrow \square(A \wedge B) \\
& \mathbf{K}:=\square(A \rightarrow B) \rightarrow(\square A \rightarrow \square B) \\
& \mathbf{N}:=\square \top \\
& \mathrm{CON}:=\neg \square \perp \\
& \mathbf{D}:=\square A \rightarrow \neg \square \neg A \\
& \mathbf{T}:=\square A \rightarrow A \\
& \text { 4:= } \square A \rightarrow \square \square A \\
& \mathbf{B}:=A \rightarrow \square \diamond A \\
& \text { DEX: }=\square A \wedge \square \neg A \rightarrow \square B \text {. }
\end{aligned}
$$

There are different systems of propositional modal logics built to model various situations. In the following table we list some simple systems which may be considered as a base for more complex systems (for further details, see Chellas, 1980).

\begin{tabular}{|cl|l|l|}
\hline & & Rules & Axioms \\
\hline $\mathrm{E}$ & classical & $\mathbf{R E}$ & \\
\hline $\mathrm{M}$ & monotonic & $\mathbf{R M}$ & $\mathrm{E} \oplus \mathbf{M}$ \\
\hline $\mathrm{MN}$ & $\mathbf{N}$-monotonic & $\mathbf{R M} \oplus \mathbf{R N}$ & $\mathrm{E} \oplus \mathbf{M} \oplus \mathbf{N}$ \\
\hline $\mathrm{R}$ & regular & $\mathbf{R R}$ & $\mathrm{E} \oplus \mathbf{M} \oplus \mathbf{C}$ \\
\hline $\mathrm{K}$ & normal & $\mathbf{R K}$ & $\mathrm{E} \oplus \mathbf{K} \oplus \mathbf{N}$ \\
& & $\mathrm{E} \oplus \mathbf{M} \oplus \mathbf{C} \oplus \mathbf{N}$ \\
\hline
\end{tabular}




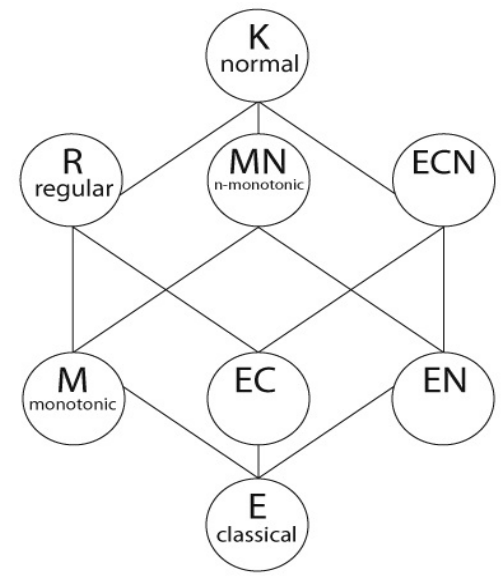

Figure 1: The lattice of non-normal propositional systems (cf. Chellas, 1980, 237).

The lattice depicted in Figure 1 illustrates the inclusion relations in non-normal systems.

In this paper we shall work with predicate extensions of $\mathbf{N}$-monotonic modal ones. For this class of logics, multi-relational semantics is as follows.

Definition 3.1 A multi-relational frame is a structure $\mathcal{F}:=\langle W, \mathcal{R}\rangle$ where $W$ is a non empty set and $\mathcal{R}$ is a set at most countable of accessibility relations.

As usual, a model is obtained by adding an evaluation function $V$ to any frame.

Truth conditions. The truth conditions for all Boolean operations are standard. Given a multi-relational a model $\mathcal{M}:=\langle\mathcal{F}, V\rangle$ and a world $w$ from $W$, the clauses to evaluate modal formulae are a direct generalisation of the standard Kripke approach: $\vDash_{w}^{V} \square A$ if and only if there exists an $R_{i}$ in $\mathcal{R}$ such that for all $v \in W\left(w R_{i} v \Rightarrow \vDash_{v}^{V} A\right)$.

Notation and abbreviations. Given a relation $R_{i}$ and a world $w$, by the symbol $R_{i}(w)$ we refer to the set of all the worlds $R_{i}$-accessible from $w$, i.e.: $R_{i}(w):=\left\{x: w R_{i} x\right\}$.

Given a model $\mathcal{M}:=\langle W, \mathcal{R}, V\rangle$ and a formula $A$, we define the truth set of $A$, in symbols $\|A\|_{V}$, as the set of all the worlds of the model in which $A$ is true, i.e.: $\|A\|_{V}:=\left\{w: \vDash_{w}^{V} A\right\}$.

Finally, let us mentions a few relevant results from Calardo and Rotolo (2014) that work on the weak formulation of multi-relational semantics based on the clause (1).

Lemma 3.2 (Schema $\mathbf{N}$ ) The schema $\mathbf{N}:=\square T$ is valid in the class of all multi-relational frames.

Lemma 3.3 (Frame characterisation of $\mathbf{C}$ ) For any multi-relational frame $\mathcal{F}$ the following holds: $\mathcal{F} \vDash \square A \wedge \square B \rightarrow \square(A \wedge B)$ iff for any world $w$, for any relation $R_{i}, R_{k}$ there exists a relation $R_{j}$ such that $R_{j}(w) \subseteq R_{k}(w) \cap R_{i}(w)$.

Theorem 3.4 (N-Monotonic logics - Soundness) Let $\mathrm{MN}_{\vdash}:=\{A \mid \mathrm{CPC} \oplus \mathbf{R E} \oplus \mathbf{M} \oplus \mathbf{N} \vdash$ $A\}$-where CPC stands for Classical Propositional Calculus-and $\mathrm{MN}_{\vDash}:=\{A \mid \vDash A\}$. Then $\mathrm{MN}_{\vdash} \subseteq \mathrm{MN}_{\models}$.

Theorem 3.5 (Completeness of MN) The logic MN is complete with respect to the class of multi-relational frames. 


\section{Quantification in N-Monotonic Modal Logics}

We have seen that a few works offer a semantic analysis of quantified non-normal modal systems using neighbourhood semantics. However, as far as we are concerned, nothing has been done so far regarding multi-relational semantics in a first order modal framework.

Let us define multi-relational structures for any quantified modal logic.

Definition 4.1 (Multi-relational frames) A multi-relational frame is a tuple $\mathcal{F}:=$ $\langle W, \mathcal{R}, D, U\rangle$ where:

- $W$ is a non empty set of worlds

- $\mathcal{R}$ is a (possibly infinite) set of binary relations over $W$

- $D$ is a function associating to each world $w \in W$ a set $D_{w}$ of individuals (the inner domain of $w$ )

- $U$ is a function associating to each world $w \in W$ a set $U_{w}$ of individuals (the outer domain of $w$ ) such that for any $w \in W, U_{w} \neq \varnothing$ and $D_{w} \subseteq U_{w}$ and if $w R v$ for some $R$, then $U_{w} \subseteq U_{v}$.

The original definition given by Kripke (1963) states that for all worlds $w, U_{w}=\cup_{v \in W} D_{v}$, setting a unique outer domain for the whole frame. However, we decided to follow the more general approach proposed by Corsi (2002):

The fact that $U_{w} \subseteq U_{v}$, if $w R v$, does not prevent $D_{w}$ from being disjoint from $D_{v}$. Kripke (1963) stipulates that for all $v \in W, U_{v}=\cup_{w \in W} D_{w}$. We generalise Kripke's original semantics by allowing $U_{w} \subseteq U_{v}$, if $w R v$, and $\bigcup_{w \in W} U_{w} \supseteq$ $\cup_{w \in W} D_{w} . \cup_{w \in W} U_{w}$ may contain individuals that never happen to come into existence. (Corsi, 2002, 1485)

Models, assignments, and the concepts of satisfaction, truth, validity are defined in the standard way.

Definition 4.2 (Multi-relational models) A multi-relational model is a tuple $\mathcal{M}:=$ $\langle W, \mathcal{R}, D, U, I\rangle$ where $\langle W, \mathcal{R}, D, U\rangle$ is a multi-relational frame and $I$ is a function $I: \mathcal{L} \times W \mapsto$ $\cup_{w \in W} U_{w}$ such that:

- $I_{w}\left(P^{n}\right) \subseteq\left(U_{w}\right)^{n}$

- $I_{w}(c) \in U_{w}$

- $I_{w}(=)=\left\{\langle d, d\rangle: d \in U_{w}\right\}$.

Definition 4.3 (Assignments) For any $w \in W$, a w-assignment $\sigma$ is a function $\sigma$ : $\operatorname{Var}(\mathcal{L}) \mapsto U_{w}$.

An $x$-variant $\tau$ of a w-assignment $\sigma$ is a $w$-assignment which may differ from $\sigma$ for the value assigned to $x$.

Notice that within the semantics framework proposed by Kripke (1963), since the outer domains are constant, any $w$-assignment $\sigma$ is also a $v$-assignment for any couple of worlds. However, it should be noticed that, here, the fact that $U_{w} \subseteq U_{v}$, if $w R v$ for some $R$, still guarantees that if two worlds $w, v$ are related by some $R$, then any $w$-assignment is also a $v$-assignment, as all the variables of the language are still mapped on individuals without gaps.

Definition 4.4 ( $\sigma$-interpretation) Given a w-assignment $\sigma$, define

(a) $I_{w}^{\sigma}(c)=I_{w}(c)$, and

(b) $I_{w}^{\sigma}(x)=\sigma(x)$.

Definition 4.5 (Truth conditions) Let $\mathcal{M}:=\langle W, \mathcal{R}, D, U, I\rangle$ be any multi-relational model, $\sigma$ any assignment, and $w \in W$. Truth evaluation clauses are as follows:

- $\mathcal{M} \models_{w}^{\sigma} P^{n}\left(t_{1}, \ldots, t_{n}\right)$ iff $\left\langle I_{w}^{\sigma}\left(t_{1}\right), \ldots, I_{w}^{\sigma}\left(t_{n}\right)\right\rangle \in I_{w}\left(P^{n}\right)$

$-\mathcal{M} \neq_{w}^{\sigma} \perp$

- $\mathcal{M} \vDash_{w}^{\sigma} \forall x A$ iff for every $x$-variant $\tau$ of $\sigma$ such that $\tau(x) \in D_{w}, \mathcal{M} \vDash_{w}^{\tau} A(x)$ 


$$
\text { - } \mathcal{M} \vDash_{w}^{\sigma} \square A \text { iff } \exists R_{i} \in \mathcal{R} \forall v \in W\left(w R_{i} v \Rightarrow \mathcal{M} \vDash_{v}^{\sigma} A\right) \text {. }
$$

Clearly, since the operators $\exists$ and $\diamond$ are defined ones, the evaluation clauses are simply: $\mathcal{M} \vDash_{w}^{\sigma} \exists x A$ iff for some $x$-variant $\tau$ of $\sigma$ such that $\tau(x) \in D_{w} \mathcal{M} \vDash_{w}^{\tau} A(x)$ and $\mathcal{M} \vDash_{w}^{\sigma} \diamond A$ iff $\forall R_{i} \in \mathcal{R}$ there is a $v \in W\left(w R_{i} v \& \mathcal{M} \vDash_{v}^{\sigma} A\right)$.

Satisfaction, Truth, Validity. A model $\mathcal{M}$ satisfies a set of formulae $\Delta$ iff for some world $w$ and some $w$-assignment $\sigma, \mathcal{M} \vDash_{w}^{\sigma} A$ for all $A \in \Delta$. A formula $A$ is true in a world $w$ of a model $\mathcal{M}, \mathcal{M} \vDash_{w} A$, iff for any $w$-assignment $\sigma, \mathcal{M} \vDash_{w}^{\sigma} A$. A formula $A$ is true in a model $\mathcal{M}, \mathcal{M} \vDash A$, iff for all $w, \mathcal{M} \vDash_{w} A$. A formula $A$ is valid on a frame $\mathcal{F}, \mathcal{F} \vDash A$, iff for any model $\mathcal{M}$ on $\mathcal{F}, \mathcal{M} \vDash A$. Given a class of frames $\mathbb{F}$, a formula $A$ is $\mathbb{F}$-valid, $\mathbb{F} \vDash A$, iff for any frame $\mathcal{F} \in \mathbb{F}, \mathcal{F} \vDash A$. $\mathcal{M}$ is a model for a logic $\mathrm{L}$ iff $\mathcal{M} \vDash A$ for all $A \in \mathrm{L}$.

Abbreviations. Given a frame $\mathcal{F}$ and a model $\mathcal{M}$ on $\mathcal{F}$, for any formula $A$,

Satisfaction: $\|A\|_{I}^{\sigma}:=\left\{w \mid \mathcal{M} \vDash_{w}^{\sigma} A\right\}$

Truth: $\|A\|_{I}:=\left\{w \mid \mathcal{M} \vDash_{w}^{\sigma} A\right.$, for any assignment $\left.\sigma\right\}$

Validity: $\|A\|:=\left\{w \mid \mathcal{F} \vDash_{w}^{\sigma} A\right.$ for any assignment $\sigma$ and any interpretation $\left.I\right\}$.

Definition 4.6 Given a multi-relational model $\mathcal{M}=\langle W, \mathcal{R}, D, U, I\rangle$, an individual constant $c$ is said to be a rigid designator iff for any $w, v$ in $W$, if there is a relation $R_{i}$ such that $\left(w R_{i} v\right)$, then $I_{w}(c)=I_{v}(c)$.

Lemma 4.7 Given a multi-relational model $\mathcal{M}=\langle W, \mathcal{R}, D, U, I\rangle$ and a w-assignment $\sigma$, if an individual constant $c$ is a rigid designator, then $\vDash_{w}^{\sigma} A(c / x)$ iff $\vDash_{w}^{\tau} A(x)$ for any $w$ assignment $\tau$ which is an $x$-variant of $\sigma$ such that $\tau(x)=I_{w}(c)$. (Cf. Corsi, 2002, Lemma 1.1).)

Proof. The proof is given by induction on the length of a formula $A$. We shall provide a proof only for the modal case, since the others are quite standard. Suppose $A$ has the form $\square B(x)$. If $\vDash_{w}^{\sigma} \square B(c / x)$ then there is a relation $R_{i}$ such that for any world $v$, if $w R_{i} v$, then $\vDash_{v}^{\sigma} B(c / x)$ and by induction hypothesis we have $\vDash_{v}^{\tau} B(x)$ where $\tau$ is an $x$-variant of $\sigma$ such that $\tau(x)=I_{v}(c)$. Since $c$ is a rigid designator by hypothesis, $I_{v}(c)=I_{w}(c), \tau$ is a $w$-assignment and hence $\vDash_{w}^{\tau} \square B(x)$.

If $\vDash_{w}^{\tau} \square B(x)$ then there is a relation $R_{i}$ such that for any world $v$, if $w R_{i} v$, then $\vDash_{v}^{\tau} B(x)$ and by induction hypothesis we have $\vDash_{v}^{\sigma} B(c / x)$ where $\tau$ is an $x$-variant of $\sigma$ such that $\tau(x)=I_{v}(c)$. Since $c$ is a rigid designator by hypothesis, $I_{v}(c)=I_{w}(c), \sigma$ is a $w$-assignment and hence $\vDash_{w}^{\sigma} \square B(c / x)$.

We assume all individual constants to be rigid designators.

Lemma 4.8 Given a multi-relational frame $\mathcal{F}$, a model $\mathcal{M}:=\langle W, \mathcal{R}, D, U, I\rangle$ on it and a world $w$, if $\sigma$ and $\tau$ are two $w$-assignments which coincide on any free variable occurring in a formula $A$, then it holds that $\mathcal{M} \vDash_{w}^{\sigma} A$ iff $\mathcal{M} \vDash_{w}^{\tau} A$.

Proof. The proof is given by induction on the length of a formula $A$. Suppose $A$ has the form $P^{n}\left(t_{1}, \ldots, t_{n}\right)$, then $\vDash_{w}^{\sigma} P^{n}\left(t_{1}, \ldots, t_{n}\right)$ if and only if $\left\langle I_{w}^{\sigma}\left(t_{1}\right), \ldots, I_{w}^{\sigma}\left(t_{n}\right)\right\rangle \in I_{w}\left(P^{n}\right)$. If $t_{i}$ is an individual constant $c$, then $I_{w}^{\sigma}(c)=I_{w}(c)=I_{w}^{\tau}(c)$. Otherwise, if $t_{i}$ is a variable $x$, $I_{w}^{\sigma}(x)=\sigma(x)=\tau(x)$ by hypothesis, hence $I_{w}^{\sigma}(x)=I_{w}^{\tau}(x)$. The other steps are straightforward.

\section{The Converse Barcan and the Ghilardi schemata}

(Stolpe, 2003) is certainly one of the most significant studies of quantified non-normal modal logics. Stolpe's aim is to find out "[...] what semantical restrictions must be imposed on a minimal model in order to validate the Barcan and the converse Barcan formulae" (Stolpe. 2003, 559, emphasis added). However, as Arló Costa pointed out: 
[...] unfortunately Stolpe does not appeal to frames in his semantics (he only uses models). So, it is obvious that there are many open questions not considered in Stolpe's paper. For example it would be nice to get frame conditions characterizing the Barcan and the Converse Barcan in this setting. (Arló-Costa, 2011. 21)

Our aim is to attack this research question in the multi-relational setting. In particular, in this section we shall propose frame conditions for $\mathbf{C B F}$ in multi-relational semantics.

Standard results in Kripke Semantics (1-relational frames) state that both BF and CBF are valid in constant domain frames (i.e., when $D_{w}$ and $U_{w}$ coincide for each $w$ ). Within first-order Kripke frames with varying domains, however, these schemata cease to be valid. Let us recall below the proof of this well known result.

Lemma 5.1 CBF is not valid in the class of multi-relational frames with varying inner domains.

Proof. The proof is trivial. Let $\mathcal{M}=\langle W, \mathcal{R}, D, U, I\rangle$ be a 1-relational model such that: $W:=\{w, v\}, \mathcal{R}:=\{R\}, R:=\{\langle w, v\rangle\}, D_{w}:=\{a, b\}, D_{v}:=\{a\}, U_{w}=U_{v}=\{a, b\}, I_{w}^{\sigma}\left(P^{1}\right)=\varnothing$ for all $\sigma$ and $I_{v}^{\sigma}\left(P^{1}\right)=D_{v}$, for $\sigma(x)=a$ (see Figure 2). Then $\|\forall x(P)\|_{I}^{\sigma}=D_{v}$ and there is a relation, i.e., $R$, such that for any world $t, w R t$ (if and) only if $\vDash_{t}^{\sigma} \forall x P$. Hence $\vDash_{w}^{\sigma} \square \forall x P$. Moreover $\vDash_{w}^{\sigma} \exists x \diamond \neg P$, as $\vDash_{v}^{\tau} \neg P(x)$ for $\tau(x)=b$.

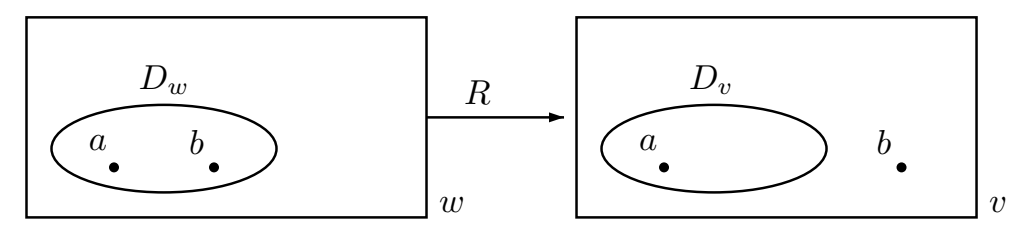

Figure 2: A frame to build a countermodel for CBF. Indeed set $I_{w}^{\sigma}\left(P^{1}\right)=\varnothing$ for all $\sigma$ and $I_{v}^{\sigma}\left(P^{1}\right)=D_{v}$, for $\sigma(x)=a$. Hence $v \vDash_{v}^{\sigma} \forall x A$.

Let us first consider the definition of local seriality, which us needed to study Barcan schemata:

Definition 5.2 (Local Seriality) Given a multi-relational frame $\mathcal{F}$, a world $w$ is locally serial iff for any relation $R_{i}$ from $\mathcal{F}$ there is a world $v$ such that $w R_{i} v$.

In what follows we shall present two different characterisation results connecting $\mathbf{C B F}$ to the concept of increasing domains. The first one (Lemma 5.3 is certainly the less intuitive, but it is needed to provide a further, more appealing, characterisation (Lemma 5.11). In fact, the lemma below provides necessary and sufficient conditions to build counter-models for CBF. After this preliminary result, we shall discuss the semantic role of $\mathbf{C B F}$ in further detail.

Lemma 5.3 (CBF Characterisation result) For any multi-relational frame $\mathcal{F}:=$ $\langle W, \mathcal{R}, D, U\rangle, \mathcal{F} \vDash \square \forall x A \rightarrow \forall x \square A$ iff $\forall w \in W$, if $w$ is locally serial, then for any a such that $a \in D_{w}$, for any relation $R_{j}$, there is some relation $R_{i}$ such that $R_{i}(w) \subseteq R_{j}(w)$ and for all $t,\left(w R_{i} t \Rightarrow a \in D_{t}\right)$.

Proof. According to the statement of the Lemma above, any counter-model for CBF should be based on a frame $\mathcal{F}$ with the following three conditions: There is a world $w \in W$, and a $w$-assignment $\sigma$ such that:

(a) BOTH $w$ is locally serial, i.e., for all $k, R_{k}(w) \neq \varnothing$

(b) AND there exists a $w$-assignment $\tau$, which is an $x$-variant of $\sigma$ such that $\tau(x) \in D_{w}$, there is a relation $R_{j}$ 
b.1 BOTH $\exists t\left(w R_{j} t \& \tau(x) \notin D_{t}\right)$, i.e., the inner domains are not increasing

b.2 AND for all the other relations $R_{i}$, IF $R_{i}(w) \subseteq R_{j}(w)$, THEN there exists some world $t_{i}$ such that $\left(w R_{i} t_{i} \& \tau(x) \notin D_{t_{i}}\right)$.

We will now show that these conditions are necessary and sufficient to build a countermodel for CBF. The idea is to provide an interpretation to make the class $R_{j}(w)$ to be exactly the truth set of $\forall x A$ for some formula $A$, so that the antecedent of CBF holds. Then, we need an individual which actually exists in $w$ and satisfies $\neg A(x)$ in some $R_{k}$-accessible state for all $k$; this would guarantee that the consequent of $\mathbf{C B F}$ is false.

Indeed, let $P$ be some unary predicate and let $I$ be an interpretation defined in the following way: For any $\vartheta$ and any world $z, z \in\|P(x)\|_{\vartheta}^{I}$ if and only if $w R_{j} z$ and $\vartheta$ is an $x$-variant of $\tau$ such that $\vartheta(x) \in D_{z}$. Thus, $R_{j}(w)=\|\forall x P(x)\|_{\tau}^{I}$ and hence $\vDash_{w}^{\tau} \square \forall x P$.

Consider now any world $z$ such that $z \notin R_{j}(w)$; We defined $I$ in such a way that for any $z$-assignment $\vartheta, \neq_{z}^{\vartheta} P(x)$ and thus this holds also for $\tau, \neq_{z}^{\tau} P(x)$. Moreover our frame conditions guarantee that for any relation $R_{i}$, if $R_{i}(w) \subseteq R_{j}(w)$, then there is some $z \in R_{i}(w)$ such that $\tau(x) \notin D_{z}$ and, following our construction of $I, \not_{z}^{\tau} P(x)$. Since $\tau(x) \in D_{w}$ by definition, it holds that $\vDash_{w}^{\tau} \exists x \diamond \neg P(x)$, thus falsifying CBF.

For the opposite direction, it is easy to see that if $\mathbf{C B F}$ does not hold on a model, its frame fulfills the conditions stated above. Assume that for some frame $\mathcal{F}, \mathcal{F} \neq \mathbf{C B F}$. Then there are an assignment $\sigma$, a valuation $I$ and a world $w$ such that $(\mathrm{a}) \vDash_{w}^{\sigma} \square \forall x A$ and (b) $\vDash_{w}^{\sigma} \exists x \diamond \neg A$ for some formula $A$. From (b) it follows that $\vDash_{w}^{\tau} \diamond \neg A(x)$ for some $\tau(x) \in D_{w}$, thus there exists some set $\left\{z_{1}, z_{2} \ldots\right\}$ such that $w R_{k} z_{k}$ for any $R_{k} \in \mathcal{R}$ and $\#_{z_{k}}^{\tau} A$. From (a) it follows that for some relation $R_{i}, R_{i}(w) \subseteq\|\forall x A\|_{I}^{\sigma}$, where $\|\forall x A\|_{I}^{\sigma}:=\left\{t \mid \vDash_{t}^{\tau}\right.$ $A(x)$ for any $\tau$ such that $\left.\tau(x) \in D_{t}\right\}$. Now, consider any relation $R_{k}$; if $R_{k}(w) \subseteq R_{i}(w)$, then by (b), $R_{k} \subseteq\|\forall x A\|_{I}^{\sigma}$ and by (a) there is some $z \in R_{k}(w)$ such that $z \notin\|A(x)\|_{I}^{\tau}$ for some $\tau(x) \in D_{w}$, thus we must conclude that $\tau(x) \notin D_{z}$.

This lemma leads to a result that is very close to that for Kripke semantics, as we shall see in what follows.

Some remarks on CBF. An immediate, yet interesting result is that the schemata $\mathbf{M}$ and $\mathbf{C B F}$ are independent, in contrast with Theorem 2.6, which establishes that, for constant non-trivial domain neighbourhood frames $\mathbf{C B F}$ is valid whenever $\mathbf{M}$ is. Indeed by the following lemma, it holds that $\mathbf{M}$ is a valid schema, whereas it is possible to build a countermodel for CBF (see Lemma 5.1.

Lemma 5.4 The schema $\mathbf{M}$ is valid in the class of all multi-relational frames.

It is hence possible to derive the following:

Corollary 5.5 The validity of $\mathbf{M}$ does not imply that of $\mathbf{C B F}$.

Lemma 5.3 offers some interesting insights about the class of CBF-frames. First of all, it states an important property about individuals and their behaviour with respect to different accessibility relations. It is well known that CBF imposes increasing inner domains on Kripke frames:

Definition 5.6 (Increasing inner domains - Kripke semantics) A relational frame $\mathcal{F}$ has increasing inner domains iff for all worlds $w, v$, if $w R v$ then $D_{w} \subseteq D_{v}$.

Theorem 5.7 (CBF characterisation in Kripke frames) CBF characterises the class of Kripke frames with increasing inner domains.

Consider any Kripke frame $\mathcal{F}:=\langle W, R, D, U\rangle$ (which is nothing but an 1-relational frame with varying domains). From the condition stated above in Lemma 5.3 we have that $\mathcal{F} \vDash$ $\square \forall x A \rightarrow \forall x \square A$ iff $\forall w \in W$, for any world $z$, if $w R z$ then for any $w$-assignment $\sigma$ such that $\sigma(x) \in D_{w}, \sigma(x) \in D_{z}$, i.e., $D_{w} \subseteq D_{z}$. Thus the conditions imposed by CBF are the usual ones: CBF is valid in those frames whose connected worlds have increasing inner domains. 
The situation within $\mathbf{N}$-monotonic logics is rather different, yet connected. The first question that comes to mind is how to generalise the concept of increasing inner domains. There are a couple of alternatives at hand, which look very close to the definition given in Kripke semantics. We might either define increasing domains in a very strong sense, by asking that all relations enjoy such property:

Definition 5.8 (General increasing inner domains) A multi-relational frame $\mathcal{F}$ has increasing inner domains iff for any couple of worlds $w, v$ for any relation $R_{i}$, if $w R_{i} v$ then $D_{w} \subseteq D_{v}$.

Alternatively, we might keep such property lighter, asking for only one relation to fulfill it:

Definition 5.9 (Restricted increasing inner domains) A multi-relational frame $\mathcal{F}$ has increasing inner domains iff for any couple of worlds $w, v$ there is at least one relation $R_{i}$, such that if $w R_{i} v$ then $D_{w} \subseteq D_{v}$.

Generally, in the multi-relational semantics scenario, CBF does not capture any of these properties. Indeed Lemma 5.3 states that if a world $w$ is locally serial, i.e., if there is access to other worlds under any relation, then each actual individual (i.e., any individual belonging to the inner domain of $w$ ) keeps being actual in a subset of every relation. So it says, somehow, that individuals are bound to "survive" under alternative relations, although not necessarily altogether. If Mary and Alex are both alive now (actual) the presence of CBF guarantees that for any relation, there is a subset of alternative possible worlds in which Mary keeps being actual, and another one in which Alex is still actual.

Logically, if we consider the big union of all the inner domains connected to $w$ by some relation $i$, the inner domain of $w$ is a subset of it, i.e.:

Lemma 5.10 If the schema $\mathbf{C B F}$ is valid on a given frame, then on any world $w$, if $w$ is locally serial, i.e., for any $i, R_{i}(w) \neq \varnothing$, then for any relation $R_{i}$

$$
D_{w} \subseteq \bigcup\left\{D_{v} \mid v \in R_{i}(w)\right\} .
$$

Proof. Assume that the property stated in this Lemma does not hold. Then there are a locally serial world $w$ and a relation $R_{i}$ such that for some individual $d \in D_{w}$, for all $v \in R_{i}(w), d \notin D_{v}$, i.e., $D_{w} \nsubseteq \bigcup\left\{D_{v} \mid v \in R_{i}(w)\right\}$. Consider an assignment $\sigma$ on any world belonging to $R_{i}(w)$ such that $\sigma(x)=d$, then for all $v \in R_{i}(w), \vDash_{v}^{\sigma} \neg \exists x(x=d)$ and then $\vDash_{w}^{\sigma} \square \forall x(x \neq d)$. By CBF we get $\vDash_{w}^{\sigma} \forall x \square(x \neq d)$, i.e., for any $w$-assignment $\tau$ such that $\tau(x) \in D_{w}, \vDash_{w}^{\tau} \square(x \neq d)$. Since $d \in D_{w}$, this holds true for $\sigma$ as well, where $\sigma(x)=d$. Hence we get that the formula $\square(d \neq d)$ holds true at $w$. This would impose that for some $j$, $R_{j}(w)=\varnothing$ which is contradictory with the assumptions.

Hence, within the same set of alternative possible worlds, there is one world in which Mary lives and another in which Alex lives, although, again, they are not bound to necessarily coincide.

A natural question is then how to force individuals to survive altogether under some relation. Well, intuitively, at first sight, it would be enough to add partial closure under intersection, i.e., the semantic property stated in Section 3 (Lemma 3.3) and characterised by schema $\mathbf{C}:=\square A \wedge \square B \rightarrow \square(A \wedge B)$. However, this would generate a normal system (see the lattice in Figure 1). Thus, in the presence of Axiom $\mathbf{C}$, the situation becomes closer to the Kripkean case. This said, Definition 5.9 seems to capture the concept of "increasing domains" better than the stronger alternative proposed. However, it turns out that the presence of $\mathbf{C}$ is not required to achieve the formerly stated property, which is actually (partially) granted by the presence of $\mathbf{C B F}$ alone:

Theorem 5.11 (CBF and Restricted Increasing Domains) For any multi-relational frame $\mathcal{F}:=\langle W, \mathcal{R}, D, U\rangle, \mathcal{F} \vDash \square \forall x A \rightarrow \forall x \square A$ iff $\forall w \in W$, if $w$ is locally serial, then for all $R_{j}$, there is some relation $R_{i}$ such that $R_{i}(w) \subseteq R_{j}(w)$ and for all worlds $v$ in $R_{i}(w)$, $D_{w} \subseteq D_{v}$. 
Proof. This is very straightforward. In fact, consider the formulation of Lemma 5.3 which states that if $w$ is locally serial, then for any $c$ such that $c \in D_{w}$, for any relation $R_{j}$, there is some relation $R_{i}$ such that $R_{i}(w) \subseteq R_{j}(w)$ and for all $t,\left(w R_{i} t \Rightarrow c \in D_{t}\right)$. Suppose the inner domain of $w$ is the set $\{a, b\}$. Then, for any $R_{j}$ there must be an $R_{i}$ such that $R_{i}(w) \subseteq R_{j}(w)$ and $a$ belongs to the inner domain of all the worlds from $R_{i}(w)$. However, since the statement of Lemma 5.3 refers to all relations and all individuals, we must consider also $R_{i}$ and $b$. Hence there is an $R_{m}$ such that $R_{m}(w) \subseteq R_{i}(w)$ and $b$ belongs to the inner domain of any world from $R_{m}(w)$. Thus, all the worlds from $R_{m}(w)$ have an inner domain containing both $a$, and $b$ and their inner domain is, therefore, a superset of $D_{w}$.

For the opposite direction, suppose by reductio that the property stated in the Lemma holds, whereas CBF does not. Let us recall the construction of a counter model for CBF as explained in Lemma 5.3 If $\mathbf{C B F}$ does not hold, there are an assignment $\sigma$, a valuation $I$ and a world $w$ such that (a) $\vDash_{w}^{\sigma} \square \forall x A$ and (b) $\vDash_{w}^{\sigma} \exists x \diamond \neg A$ for some formula $A$. From (b) it follows that $\vDash_{w}^{\tau} \diamond \neg A(x)$ for some $\tau(x) \in D_{w}$, thus for any $R_{k} \in \mathcal{R}$, there is a world $z_{k}$ such that $w R_{k} z_{k}$ and $\sharp_{z_{k}}^{\tau} A$. From (a) it follows that for some relation $R_{i}, R_{i}(w) \subseteq\|\forall x A\|_{I}^{\sigma}$, where $\|\forall x A\|_{I}^{\sigma}:=\left\{t \mid \underset{F_{t}^{\tau}}{F^{\tau}} A(x)\right.$ for any $\tau$ such that $\left.\tau(x) \in D_{t}\right\}$. Now consider the relation $R_{i}(w)$. The statement of this Lemma says that (c) there must be a relation $R_{j}$ such that $R_{j}(w) \subseteq R_{i}(w)$ and for all worlds $v$ in $R_{i}(w), D_{w} \subseteq D_{v}$. Moreover, by (a), since $R_{j}(w) \subseteq R_{i}(w)$, for any $v \in R_{j}(w)$ it holds that $\vDash_{v}^{\sigma} \forall x A$. However by (b) for some $z \in R_{j}(w)$, $\neq_{z}^{\tau} A(x)$ and $\tau(x) \in D_{w}$. But by (c) $D_{w} \subseteq D_{z}$, hence we reach a contradiction.

By all means, these results are much more specific than those proposed by Stolpe $(2003)$, as nothing is known about inner domains of related worlds. In fact, by translating in terms of multi-relational semantics Stolpe (2003)'s CUPO condition (see Theorem 2.9p it is not hard to prove a weaker correspondence result for CBF in multi-relational models.

Lemma 5.12 (CUPO models) For any multi-relational model $\mathcal{M}:=\langle W, \mathcal{R}, D, U, I\rangle, \mathcal{M} \vDash$ $\square \forall x A \rightarrow \forall x \square A$ if and only if for any world $w$ the following holds: given a $w$-assignment $\sigma$, if there is a relation $R_{i}$ such that $R_{i}(w) \subseteq\|\forall x A\|_{I}^{\sigma}$, then for all $x$-variant $\tau$ of $\sigma$ such that $\tau(x) \in D_{w}$ there is some $j$ such that $R_{j}(w) \subseteq\|A(x)\|_{I}^{\tau}$.

Proof. For the left arrow, suppose there are a model $\mathcal{M}:=\langle W, \mathcal{R}, V\rangle$ and a $\sigma$ such that $\vDash_{w}^{\sigma} \forall x A$. Hence, for some $i, R_{i}(w) \subseteq\|\forall x A\|_{w}^{\sigma}$. By hypothesis for all $x$-variant $\tau$ of $\sigma$ such that $\tau(x) \in D_{w}$ there is some $j$ such that $R_{j}(w) \subseteq\|A(x)\|_{I}^{\tau}$, thus ensuring $\vDash_{w}^{\tau} \square A(x)$ and hence $\models_{w}^{\sigma} \forall x \square A(x)$. The other version can be proved accordingly by contraposition.

The reader may easily notice that Stolpe's result does not say anything regarding the inner structure of frames, but it is strictly related to specific interpretations. On the other hand, a frame characterisation result is based on structural properties on domains of individuals (here also interplaying with relations), as analysed in the literature on quantified modal logics, where CBF is classically associated with the condition of increasing domains of individuals (Garson, 2001, Fitting and Mendelsohn, 1998, Corsi, 2002, Garson, 2005, Brauner and Ghilardi., 2007; Gabbay et al., 2009, Goldblatt, 2011).

Mirroring Kripke semantics, $\mathbf{C B F}$ and the Ghilardi schema $\exists x \square A \rightarrow \square \exists x A$ (GF) are semantically equivalent:

Lemma 5.13 CBF and GF are semantically equivalent

Proof. To show this result it is enough to check that GF characterises the class of CBFframes.

$(\Rightarrow)$ According to the statement of Lemma 5.3 any counter-model for CBF must be based on a frame $\mathcal{F}$ with the following three conditions:

there is a world $w \in W$, and a $w$-assignment $\sigma$ such that:

(a) BOTH $w$ is locally serial, i.e., for all $k, R_{k}(w) \neq \varnothing$

(b) AND there exists an individual $a, a \in D_{w}$, and there is a relation $R_{j}$

(b.1) BOTH $\exists t\left(w R_{j} t \& a \notin D_{t}\right)$, i.e., the inner domains are not increasing 
(b.2) AND for all the other relations $R_{i}$, IF $R_{i}(w) \subseteq R_{j}(w)$, THEN there exists some world $t_{i}$ such that $\left(w R_{i} t_{i} \& a \notin D_{t_{i}}\right)$.

Let us sketch a valuation to build a counter-model for GF. Let $A$ be a unary predicate, take any $w$-assignment $\tau$ and set $I$ as follows:

(a) IF $\tau(x) \neq a$, then $\|A(x)\|_{I}^{\tau}=W$;

(b) OTHERWISE if $\tau(x)=a$, then $\|A(x)\|_{I}^{\tau}=W-R_{i}(w)$.

From (b) it follows that $R_{i}(w) \subseteq\|\neg A(x)\|_{I}^{\tau}$, hence $\vDash_{w}^{\sigma} \exists x \square \neg A(x)$.

Let us turn our attention to (a). Any world $v$ which is not $R_{i}$-seen by $w$ is such that for any assignment $\tau, \vDash_{v}^{\tau} A(x)$ and hence $\vDash_{v}^{\sigma} \forall x A(x)$ for all $\sigma$. Concerning $R_{i}$ the situation is the following: the fact that $\exists t\left(w R_{j} t \& a \notin D_{t}\right)$ guarantees that $\vDash_{t}^{\sigma} \forall x A(x)$, whereas the fact that for all the other relations $R_{i}$, IF $R_{i}(w) \subseteq R_{j}(w)$, THEN there exists some world $t_{i}$ such that $\left(w R_{i} t_{i} \& a \notin D_{t_{i}}\right)$, guarantees the fact that there is always a world $z$ in $R_{j}(w)$ such that $\vDash_{z}^{\sigma} \forall x A(x)$. This observation, together with the fact that $w$ is locally serial by assumption, makes sure that $\vDash_{w}^{\sigma} \diamond \forall x A(x)$, thus disproving an instance of GF.

\section{Completeness Results 5}

\subsection{The System $Q_{=}^{\circ} \cdot M N$}

Here we present an axiomatic system extending MN with predicate logic, which is based on free quantified modal logic (see Corsi 2002, 1498). The system $Q_{=}^{\circ} . M N$ (Free Quantified $\mathrm{N}$-monotonic modal logic) contains the following axioms and inference rules:
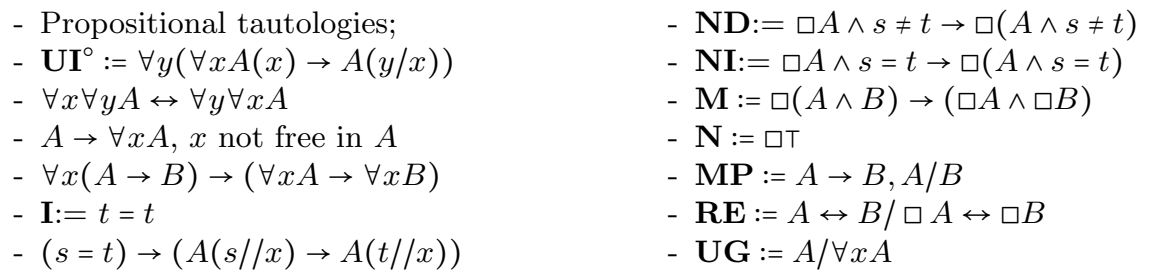

Some remarks on $\mathrm{Q}_{=}^{\circ} . \mathrm{MN}$. Besides the propositional part, axiom schemata for the basic predicate part (not considering identity) are those originally proposed by Kripke (1963) (see Corsi, 2002) plus $\forall x \forall y A \leftrightarrow \forall y \forall x A$, which is conceptually harmless but needed to ensure completeness results (see Goldblatt, 2011). The language includes the identity symbol =, which makes the logic very expressive. Notice that the expression $A(t / / s)$ denotes that some (all, none) free occurrences of $s$ are replaced by $t$, whereas $A\left(s_{1} \ldots, t / s_{i}, \ldots s,\right)$ stands for $A\left(s_{1} \ldots, s_{i} \ldots s_{n}\right)\left(t / s_{i}\right)$. (see Corsi $(2002)$ ). In quantified modal logic with identity, logicians usually consider whether the following schemata are to be valid (for a philosophical discussion, see Kripke, 1980):

$$
t=s \rightarrow \square(t=s) \quad t \neq s \rightarrow \square(t \neq s)
$$

Here, we consider a version of them that can also capture some quite restricted versions of $\mathbf{C}$ : indeed, ND and NI state that it is possible to modally aggregate two formulae when one is any $A$ (and thus $A$ can also be a $\square$ formula) and the other is either $t=s$ or $t \neq s$; this does not in general entail C. Notice that the schema $\mathbf{N I}^{*}:=t=s \rightarrow \square(t=s)$ is derivable within this system. Finally, it is worth noting that we will make an essential use of the identity symbol and schemata $\mathbf{N I}$ and $\mathbf{N I}^{*}$ to ensure completeness of the system $\mathbf{Q}_{=}^{\circ} \cdot \mathbf{M N} \oplus \mathbf{C B F}$, namely, when we add CBF. We will show later why this is technically needed.

\footnotetext{
${ }^{5}$ We wish to thank Gabriele Tassi for the fruitful discussions and the useful insights he provided concerning the issues discussed in this section.
} 
Given a language $\mathcal{L}$, we shall henceforth refer to the set of its individual constants with the notation $\operatorname{Const}(\mathcal{L})$.

Theorem 6.1 (Soundness) The system $\mathrm{Q}_{=}^{\circ} . \mathrm{MN}$ is sound with respect to the class of all multi-relational frames with varying inner domains.

Proof. The proof is standard and it is carried out by induction on the length of $\mathcal{D}$, where $\mathcal{D}:=\mathcal{D}_{1}, \ldots, \mathcal{D}_{n}$ is a deduction in $n$ steps in the axiomatic system $Q_{=}^{\circ} . \mathrm{MN}$ with $A=\mathcal{D}_{n}$, i.e., $A \in Q_{=}^{\circ} \cdot \mathrm{MN}_{\vdash}$.

(a) If $k=1, A$ is an axiom. Let us consider just a few cases. For the propositional schemata, please refer to Theorem 3.4 If $A$ has the form $\forall y(\forall x B(x) \rightarrow B(y / x))$, then assume by reductio that there are a multi-relational frame $\mathcal{F}$, a model $\mathcal{M}=\langle W, \mathcal{R}, D, U, I\rangle$ on $\mathcal{F}$, a world $w$ and a $w$-assignment $\sigma$ such that $\mathcal{M} \neq_{w}^{\sigma} \forall y(\forall x B(x) \rightarrow B(y / x))$. Then there is a $w$-assignment $\tau$ which is an $y$-variant of $\sigma$ such that $\mathcal{M} \neq_{w}^{\tau} \forall x B(x) \rightarrow B(y / x)$ and $\tau(y) \in D_{w}$. Hence $\mathcal{M} \vDash_{w}^{\tau} \forall x B(x)$ and $\mathcal{M} \neq_{w}^{\tau} B(y / x)$ and $\tau(y) \in D_{w}$. It follows that for any $w$-assignment $\vartheta$, where $\vartheta$ is an $x$-variant of $\tau$ such that $\vartheta(x) \in D_{w}, \mathcal{M} \vDash_{w}^{\vartheta} B(x)$ and $\mathcal{M} \neq_{w}^{\tau} B(y / x)$ for $\tau(y) \in D_{w}$. Since one must consider all the $x$-variants of $\tau$ which map $x$ to an element of the inner domain of $w$ and since $\tau(y) \in D_{w}$, there is an assignment $\vartheta^{\star}$ such that it is an $x$-variant of $\tau, \vartheta^{\star}(x)=\tau(y), \mathcal{M} \vDash_{w}^{\vartheta^{\star}} B(x)$ and $\mathcal{M} \neq_{w}^{\tau} B(y / x)$.

If $A$ has the form $B \rightarrow \forall x B$ for $x$ not free in $B$, then suppose by reductio that there are a multi-relational frame $\mathcal{F}$, a model $\mathcal{M}=\langle W, \mathcal{R}, D, U, I\rangle$ on $\mathcal{F}$, a world $w$ and a $w$-assignment $\sigma$ such that $\mathcal{M} \neq_{w}^{\sigma} B \rightarrow \forall x B$, thus $\mathcal{M} \vDash_{w}^{\sigma} B$ and $\mathcal{M} \vDash_{w}^{\sigma} \forall x B$, i.e., $\mathcal{M} \vDash_{w}^{\sigma} \exists x \neg B$. From this it follows that $\mathcal{M} \neq_{w}^{\tau} B$ for some $x$-variant $\tau$ of $\sigma$ such that $\tau(x) \in D_{w}$. Since $x$ is not free in $B, \sigma$ and $\tau$ coincide on any free variable occurring in $B$, hence, by Lemma 4.8 it holds that $\mathcal{M} \vDash_{w}^{\sigma} B$ and $\mathcal{M} \neq_{w}^{\sigma} B$.

If $A$ has the form $\forall x(B \rightarrow C) \rightarrow(\forall x B \rightarrow \forall x C)$, then suppose by reductio that there are a multi-relational frame $\mathcal{F}$, a model $\mathcal{M}=\langle W, \mathcal{R}, D, U, I\rangle$ on $\mathcal{F}$, a world $w$ and a $w$-assignment $\sigma$ such that $\vDash_{w}^{\sigma} \forall x(B \rightarrow C), \vDash_{w}^{\sigma} \forall x B$ and $\vDash_{w}^{\sigma} \exists x \neg C$. Thus (i) $\mathcal{M} \vDash_{w}^{\tau} B \rightarrow C$ for any $x$-variant $\tau$ of $\sigma$ such that $\tau(x) \in D_{w}$; (ii) $\mathcal{M} \vDash_{w}^{\vartheta} B$ for any $x$-variant $\vartheta$ of $\sigma$ such that $\vartheta(x) \in D_{w}$; (iii) $\mathcal{M} \neq_{w}^{v} C$ for some $x$-variant $v$ of $\sigma$ such that $v(x) \in D_{w}$. From (i) it follows that for any $x$-variant $\tau$ of $\sigma$ such that $\tau(x) \in D_{w}$, either $\mathcal{M} \neq_{w}^{\tau} B$, contradicting (ii), or $\mathcal{M} \vDash_{w}^{\tau} C$, contradicting (iii).

If $A$ has the form $\square B \wedge s=t \rightarrow \square(B \wedge s=t)$, then assume that there are a model, a world and an assignment such that $\vDash_{w}^{\sigma} \square B \wedge s=t$. This entails the existence of a relation $R_{i}$ such that $R_{i}(w) \subseteq\|B\|_{I}^{\sigma}$. Take any $v \in R_{i}(w)$. If $t$ (or $s$ ) is an individual constant, then $I_{w}^{\sigma}(t)=I_{v}^{\sigma}(t)$, since constants are rigid designators; on the other hand if $t$ (or $s$ ) is a variable, than, since $U_{w} \subseteq U_{v}$, it holds that $I_{w}^{\sigma}(t)=\sigma(t)=I_{v}^{\sigma}(t)$. Thus $\vDash_{w}^{\sigma} \square(B \wedge(t=s))$.

(b) If $k=n+1$, then $A$ is an axiom (see previous cases) or it has been obtained either via MP, or via RM, or else via UG. For the first two cases, please refer to Theorem 3.4 Let us focus on the latter case. If $A$ has been obtained via the rule $\mathbf{U G}$, it has the form $\forall x B$ and it has been derived applying $\mathbf{U G}$ to a formula $B$. By $\mathrm{IH}, \vDash_{w}^{\sigma} B$ for any valuation $I$, any world $w$ and any assignment $\sigma$. In particular this holds true for any assignment $\tau$ which is an $x$-variant of $\sigma$ such that $\tau(x) \in D_{w}$ and hence $\vDash_{w}^{\sigma} \forall x B$.

\subsection{Some Auxiliary Results}

Following Corsi (2002), let us establish some auxiliary results.

Definition 6.2 Let $\mathrm{L}$ be a logic on the language $\mathcal{L}, \Delta \subseteq \mathrm{L}$ and $Q \subseteq \operatorname{Const}(\mathcal{L})$.

- $\Delta$ is L-consistent iff $\Delta \nvdash_{\mathrm{L}} \perp$.

- $\Delta$ is L-deductively closed iff for any sentence $A$ of $\mathcal{L}, \Delta \vdash \mathrm{L} A$ iff $A \in \Delta$. 
- $\Delta$ is L-complete iff for any sentence $A$ of $\mathcal{L}$, either $A \in \Delta$ or $\neg A \in \Delta$.

- $\Delta$ is $\mathrm{L}$-maximal iff $\Delta$ is $\mathrm{L}$-consistent and $\mathrm{L}$-complete.

- $\Delta$ is $Q$-universal iff if $\forall x A(x) \in \Delta$, then $A(c / x) \in \Delta$, for all individual constants $c \in Q$. $\Delta$.

- $\Delta$ is $Q$-existential iff if $A(c / x) \in \Delta$ for some individual constant $c \in Q$, then $\exists x A(x) \in$

- $\Delta$ is $Q$-inductive iff if $A(c / x) \in \Delta$ for all individual constants $c \in Q$, then $\forall x A(x) \in \Delta$.

- $\Delta$ is $Q$-rich iff if $\exists x A(x) \in \Delta$ for some individual constant $c \in Q$, then $A(c / x) \in \Delta$.

- $\Delta$ is $\mathrm{L}$-saturated iff if $\Delta$ is $\mathrm{L}$-maximal and for some $Q \subseteq \operatorname{Const}(\mathcal{L}), \Delta$ is $Q$-universal and $Q$-rich.

Lemma 6.3 Let $L \subseteq Q_{=}^{\circ}$.MN be a logic on $\mathcal{L}$. Let $C$ be a denumerable set of individual constants not occurring in $\mathcal{L}$, let $\mathcal{L}^{C}$ be the language obtained adding $C$ to $\operatorname{Const}(\mathcal{L})$ and $\mathrm{L}^{C}$ be the logic on $\mathcal{L}^{C}$.

i. If $\vdash_{\mathrm{L}^{C}} A\left(c_{1}, \ldots, c_{n}\right)$, then $\vdash_{\mathrm{L}^{C}} A\left(x_{1} / c_{1}, \ldots, x_{n} / c_{n}\right)$ where $x_{1}, \ldots, x_{n}$ are variables not occurring in $A\left(c_{1}, \ldots, c_{n}\right)$.

ii. If $\vdash_{\mathrm{L}^{C}} A$ and no constants of $C$ occur in $A$, then $\vdash_{\mathrm{L}} A$.

iii. If $\Delta$ is an $\mathrm{L}$-consistent set of sentences and no constant from $C$ occurs in $\Delta$, then $\Delta$ is $\mathrm{L}^{C}$-consistent. (Cf. (Corsi, 2002, Lemma 1.6))

Lemma 6.4 Given an L-maximal set $\Delta$ of sentences in $\mathcal{L}$ and $Q \subseteq \operatorname{Const}(\mathcal{L})$, if $\Delta$ is $Q$-universal then $\Delta$ is Q-existential.(Cf. (Corsi, 2002, Lemma 1.11))

Proof. Assume $A(c / x) \in \Delta$ for some individual constant $c \in \operatorname{Const}(\mathcal{L})$ and $\exists x A \notin \Delta$. Since $\Delta$ is L-maximal, $\neg \exists x A(x) \in \Delta$ and hence $\forall x \neg A(x) \in \Delta$. Thus, since $\Delta$ is $Q$-universal by definition, $\neg A(c / x) \in \Delta$ and hence $\perp \in \Delta$, contradicting the consistency of $\Delta$.

Lemma 6.5 (Lindenbaum's Lemma) Given a logic L, if $\Delta$ is a L-consistent set of formulae, then there is a L-maximal set $\Delta^{+}$such that $\Delta \subseteq \Delta^{+}$.

Lemma 6.6 Let $\Delta$ be an L-consistent set of sentences of $\mathcal{L}$. Then for some not-empty denumerable set $C$ of new constants, there is a set $\Pi$ of sentences of $\mathcal{L}^{C}$ such that $\Delta \subseteq \Pi$, $\Pi$ is $\mathrm{L}^{C}$-maximal, $\Pi$ is $Q$-universal and $Q$-rich for some set $Q \subseteq \operatorname{Const}\left(\mathcal{L}^{C}\right)$. (cf. (Corsi) 2002, Lemma 1.16))

\subsection{Canonical Models}

In order to define canonical models for a language with identity, we need to introduce a binary equivalence relation on individual constants:

$$
a \sim b \text { if and only if }(a=b) \in w .
$$

Any individual constant $c$ may be interpreted on its equivalence class $[c]$, where $[c]:=\{a \mid$ $c \sim a\}$.

Definition 6.7 (Non-normal canonical model for $\mathrm{Q}_{=}^{\circ} . \mathrm{MN}$ ) Let $\mathrm{L} \supseteq \mathrm{Q}_{=}^{\circ} . \mathrm{MN}$ be a logic based on $\mathcal{L}$. Let $Q$ be a set of constants with cardinality $\aleph_{0}$ such that $Q \supset \operatorname{Const}(\mathcal{L})$ and $|Q-\operatorname{Const}(\mathcal{L})|=\aleph_{0}$. A non-normal canonical model for $\mathrm{L}$ is a tuple $\mathcal{M}=\langle W, \mathcal{R}, D, I\rangle$ such that:

- $W$ is the class of all $\mathrm{L}_{w}$-saturated sets of sentences $w$, where $\mathcal{L}_{w}=\mathcal{L}^{S}$ for some set $S$ of constants such that $\operatorname{Const}\left(\mathcal{L}^{S}\right) \neq \varnothing, S \subset Q$ and $\left\|Q-\operatorname{Const}\left(\mathcal{L}^{S}\right)\right\|=\aleph_{0}$ (where $\mathcal{L}^{S}$ is defined as in Lemma 6.3).

- For any formula $A \in F m a(\mathcal{L})$ let $R_{A}$ be a binary relation over $W$. For all $w, v \in W$, $w R_{A} v$ iff $\square A \in w \Rightarrow A \in v$ and for any constant $c \in \operatorname{Const}\left(\mathcal{L}_{w}\right),[c]_{w}=[c]_{v}$, where $[c]_{v}=$ $\left\{b \in \operatorname{Const}\left(\mathcal{L}_{v}\right):(b=c) \in v\right\}$. The set of relations $\mathcal{R}$ is the collection of all such relations.

- $D_{w}=\left\{[c]_{w}: \exists x(x=c) \in w\right\}$.

- $U_{w}=\left\{[c]_{w}: c \in \operatorname{Const}\left(\mathcal{L}_{w}\right)\right\}$.

- $I_{w}(c)=[c]_{w}$.

- $I_{w}\left(P^{n}\right)=\left\{\left\langle\left[c_{1}\right]_{w}, \ldots,\left[c_{n}\right]_{w}\right\rangle: P^{n}\left(c_{1}, \ldots, c_{n}\right) \in w\right\}$. 
Notice that this definition implies that whenever a formula $\square A$ does not belong to a state $w$, the relation associated to $A$ for $w$ would be $R_{A}(w)=W$, i.e., the whole universe.

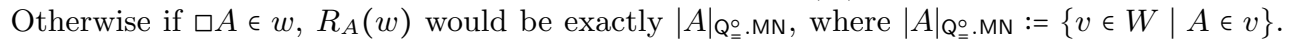
Moreover, it is important to notice that the frame of a $\mathrm{Q}_{=}^{\circ} \cdot \mathrm{MN}$-canonical model is not always generally serial. Indeed it allows the presence of empty relations for any world, and hence the schema $\mathbf{C O N}:=\diamond \top$ is not valid on the canonical frame.

Lemma 6.8 (Existence lemma) Given a canonical model $\mathcal{M}$ for $\mathrm{Q}_{=}^{\circ} . \mathrm{MN}$, for any $w \in W$, if $\diamond A \in w$, then for any formula $B \in F m a(\mathcal{L})$ there is a set $v$ such that:

1. $v$ belongs to the base set of $\mathcal{M}$, i.e., in the set of possible worlds $W$

2. $A \in v$

3. If $\square B \in w$ then $B \in v$

4. $\operatorname{Const}\left(\mathcal{L}_{w}\right) \subseteq \operatorname{Const}\left(\mathcal{L}_{v}\right)$

5. for any individual constant $c \in \operatorname{Const}\left(\mathcal{L}_{w}\right),[c]_{w}=[c]_{v}$.

Proof. Below we shall define a procedure to construct any such $v$ for any formula $B$.

Let $C$ be a denumerable set of constants which do not belong to $\mathcal{L}_{w}$ and let $\mathcal{L}_{w}^{C}=\mathcal{L}_{w} \cup C$. Let $H_{1}, H_{2}, H_{3}, \ldots$ be an enumeration of all the existential formulae of $\mathcal{L}_{w}^{C}$ where any formula occurs infinitely often.

Let $\Gamma$ be a chain of sets as defined below:

1. $\Gamma_{0}$ :

(1.a) If $\square B \in w$, then $\Gamma_{0}:=\{B\} \cup\{A\}$

(1.b) otherwise, $\Gamma_{0}:=\{A\}$.

2. $\Gamma_{1}:=\Gamma_{0} \cup\{(a=b) \mid(a=b) \in w\}$

3. Let $\Gamma_{n}$ be already defined for $1 \leq n$ and let $H_{n+1}=\exists x F(x)$. The set $\Gamma_{n+1}$ is defined as:

(3.a) If $\exists x F(x)$ contains at least one constant $c$ such that $c \notin \operatorname{Const}\left(\Gamma_{n}\right)$, then $\Gamma_{n+1}:=$ $\Gamma_{n}$.

(3.b) otherwise, if any constant occurring within $\exists x F(x)$ is already in $\Gamma_{n}$, then there are a few cases:

(3.b.1) If $\Gamma_{n} \cup\{\exists x F(x)\}$ is $\mathrm{L}_{\mathrm{w}}^{\mathrm{C}}$-consistent, then

(3.b.1.1) $\Gamma_{n+1}:=\Gamma_{n} \cup\{\exists x F(x)\} \cup\{F(b / x)\}$, where $b \in \operatorname{Const}\left(\Gamma_{n}\right)$ and $\Gamma_{n} \cup$ $\{F(b / x)\}$ is $\mathrm{L}_{\mathrm{w}}^{\mathrm{C}}$-consistent;

(3.b.1.2) $\Gamma_{n+1}:=\Gamma_{n} \cup\{\exists x F(x)\} \cup\{F(c / x)\} \cup\left\{(c \neq b) \mid b \in \operatorname{Const}\left(\Gamma_{n}\right)\right\}$, if $\Gamma_{n} \cup\{F(b / x)\}$ is not $\mathrm{L}_{\mathrm{w}}^{\mathrm{C}}$-consistent for any $b \in \operatorname{Const}\left(\Gamma_{n}\right)$, and $c \in C$ is a constant not occurring in $\Gamma_{n}$.

(3.b.2) Otherwise if $\Gamma_{n} \cup\{\exists x F(x)\}$ is not $\mathrm{L}_{\mathrm{w}}^{\mathrm{C}}$-consistent, then $\Gamma_{n+1}=\Gamma_{n}$.

4. $\Gamma=\bigcup_{n \in \mathcal{N}}\left(\Gamma_{n}\right)$.

In order to show that $\Gamma$ is $\mathrm{L}_{\mathrm{w}}^{\mathrm{C}}$-consistent, we have to check that for any $n, \Gamma_{n}$ is $\mathrm{L}_{\mathrm{w}^{-}}^{\mathrm{C}}$ consistent.

1. $\Gamma_{0}$ is consistent. Indeed:

(1.a) If $\Gamma_{0}:=\{A, B\}$ and $\square B \in w$, then suppose $\vdash_{\mathrm{MN}} A \wedge B \rightarrow \perp$, then $\vdash_{\mathrm{MN}} B \rightarrow(A \rightarrow \perp)$, $\vdash$ MN $B \rightarrow \neg A$ and $\vdash$ MN $\square B \rightarrow \square \neg A$ by the $\mathbf{R M}$ rule. By definition, it follows that $w \vdash \mathrm{MN} \square \neg A, \neg \diamond A \in w$, leading to a contradiction as $w$ is consistent by assumption.

(1.b) If $\Gamma_{0}:=\{A\}$, then assume it is not consistent and hence $\vdash_{\mathrm{MN}} A \rightarrow \perp$, i.e., $\vdash$ MN $\neg A$. Thus by the necessitation rule we have $\vdash_{\mathrm{MN}} \square \neg A$ and this implies that $\neg \diamond A \in w$ and hence $\diamond A \notin w$, which is in contradiction with our hypothesis.

2. $\Gamma_{1}$ is consistent. Indeed assume it is not. There are two cases: 
(2.a) $\Gamma_{0}:=\{A, B\}$ and $\square B \in w$, then $\vdash_{\mathrm{MN}} B \rightarrow(a=b \rightarrow(A \rightarrow \perp))$ for some $a=b \in w$. Then $\vdash$ MN $B \rightarrow(a=b \rightarrow \neg A)$, and $\vdash_{\mathrm{MN}} \square B \rightarrow \square((a=b) \rightarrow \neg A)$ by the RM rule. By definition of $w$ and MP, it follows that $w \vdash \mathbf{M N} \square((a=b) \rightarrow \neg A)$. Thus by the $\mathbf{R M}$ rule $\vdash_{\mathrm{MN}} \square(a=b) \rightarrow \square \neg A$. But $\square(a=b) \in w$ by the $\mathbf{N I}^{*}$ schema, hence $\neg \diamond A \in w, \diamond A \notin w$, which contradicts the hypothesis.

(2.b) If $\Gamma_{0}:=\{A\}$, then $\vdash_{\mathrm{MN}}(a=b) \rightarrow A \rightarrow \perp$, i.e., $\vdash_{\mathrm{MN}}(a=b) \rightarrow \neg A$ for some $(a=b) \in w$. Thus by the $\mathbf{R M}$ rule $\vdash$ MN $\square(a=b) \rightarrow \square \neg A$. But $\square(a=b) \in w$ by the NI schema, hence $\neg \diamond A \in w, \diamond A \notin w$, which contradicts the hypothesis.

3. $\Gamma_{n+1}$ is also consistent, in fact:

(3.a) If $\exists x F(x)$ contains at least one constant $c$ such that $c \notin \operatorname{Const}\left(\Gamma_{n}\right)$, then $\Gamma_{n+1}=$ $\Gamma_{n}$ and thus it is $\mathrm{L}_{\mathrm{w}}^{\mathrm{C}}$-consistent by the Inductive Hypothesis (IH henceforth).

(3.b) If any constant occurring within $\exists x F(x)$ is already in $\Gamma_{n}$, then

(3.b.1) If $\Gamma_{n} \cup\{\exists x F(x)\}$ is $\mathrm{L}_{\mathrm{w}}^{\mathrm{C}}$-consistent, then

(3.b.1.1) The set $\Gamma_{n+1}=\Gamma_{n} \cup\{\exists x F(x)\} \cup\{F(b / x)\}$ (where $b \in \operatorname{Const}\left(\Gamma_{n}\right)$ and $\Gamma_{n} \cup\{F(b / x)\}$ is $\mathrm{L}_{\mathrm{w}}^{\mathrm{C}}$-consistent) is consistent by construction and $\mathrm{IH}$;

(3.b.1.2) Assume $\Gamma_{n+1}=\Gamma_{n} \cup\{\exists x F(x)\} \cup\{F(c / x)\} \cup\left\{(c \neq b) \mid b \in \operatorname{Const}\left(\Gamma_{n}\right)\right\}$, if $\Gamma_{n} \cup\{F(b / x)\}$ is not $\mathrm{L}_{w}^{\mathrm{C}}$-consistent for any $b \in \operatorname{Const}\left(\Gamma_{n}\right)$, and $c \in C$ is a constant not occurring in $\Gamma_{n}$. We know that the set $\Gamma_{n} \cup\{\exists x F(x)\}$ is consistent by hypothesis. First of all, let us show that $\Gamma_{n} \cup\{F(c / x)\}$ is $\mathrm{L}_{w}^{\mathrm{C}}$-consistent. Assume by reductio that $\Gamma_{n} \cup\{F(c / x)\}$ is not $\mathrm{L}_{\mathrm{w}}^{\mathrm{C}}$-consistent. Then there are sentences $\left\{D_{1}, \ldots, D_{k}\right\} \in \Gamma_{n}$ such that $\vdash_{L_{w}^{C}} D_{1} \wedge \ldots \wedge D_{k} \wedge$ $F(c / x) \rightarrow \perp$

$\Gamma_{n} \vdash_{\mathrm{L}_{\mathrm{w}}^{\mathrm{C}}} \neg(F(c / x)$

$\Gamma_{n} \vdash_{\mathrm{L}} \rightarrow F(y / c)$

$\Gamma_{n} \vdash_{L_{w}^{c}} \forall y \neg F(y / c)$

$\Gamma_{n} \vdash_{\mathrm{L}_{w}^{c}}^{c} \neg \exists y F(y)$

contrary to the fact that $\Gamma_{n} \cup\{\exists x F(x)\}$ is $\mathrm{L}_{\mathrm{w}}^{\mathrm{C}}$-consistent by hypothesis, thus $\Gamma_{n} \cup\{F(c / x)\}$ is $\mathrm{L}_{w}^{\mathrm{C}}$-consistent.

Assume by reductio that $\Gamma_{n+1}$ is not $L_{w}^{C}$-consistent. Hence for some finite set of individual constants $\left\{b_{1}, \ldots, b_{h}\right\} \subseteq \operatorname{Const}\left(\Gamma_{n}\right)$,

$\Gamma_{n} \vdash_{L_{\mathrm{w}}} F(c / x) \wedge\left(c \neq b_{1} \wedge \ldots \wedge c \neq b_{h}\right) \rightarrow \perp$

$\Gamma_{n} \vdash_{\mathrm{L} c \mathrm{w}} F(c / x) \rightarrow \neg\left(c \neq b_{1} \wedge \ldots \wedge c \neq b_{h}\right)$

$\Gamma_{n} \vdash_{L^{c}} F(c / x) \rightarrow\left(c=b_{1} \vee \ldots \vee c=b_{h}\right)$

$\Gamma_{n} \cup\{F(c / x)\} \vdash_{\mathrm{L}_{w}^{C}} c=b_{1} \vee \ldots \vee c=b_{h}$

hence for some $i, 1 \leq i \leq h, \Gamma_{n} \cup\left\{F\left(b_{i} / x\right)\right\}$ is $\mathrm{L}_{\mathrm{w}}^{\mathrm{C}}$-consistent, in contradiction with the assumption that there is no constant $b \in \operatorname{Const}\left(\Gamma_{n}\right)$ such that $\Gamma_{n} \cup\{F(b / x)\}$ is $\mathrm{L}_{w}^{C}$-consistent. Therefore $\Gamma_{n+1}$ is $\mathrm{L}_{w}^{\mathrm{C}}$-consistent.

(3.b.2) If $\Gamma_{n} \cup\{\exists x F(x)\}$ is not $\mathrm{L}_{w}^{\mathrm{C}}$-consistent, then $\Gamma_{n+1}=\Gamma_{n}$ and it is thus consistent by IH.

4. Since $\Gamma$ is the big union of a chain of consistent sets, then $\Gamma$ is $L_{w}^{C}$-consistent.

Let $Q=$ Const $(\Gamma)$. We start by showing that

(*) For any existential formula $\exists x F(x)$ of $\mathcal{L}_{w}^{Q}$ there is some $\Gamma_{k}$ such that either $\exists x F(x) \in \Gamma_{k+1}$ or $\Gamma_{k} \cup\{\exists x F(x)\}$ is $\mathcal{L}_{w}^{C}$-inconsistent.

Let $c_{1}, \ldots, c_{j}$ be all the constants occurring in $\exists x F(x)$. Since $\exists x F(x) \in \mathcal{L}_{w}^{Q},\left\{c_{1}, \ldots, c_{j}\right\} \subseteq Q$, hence for some $j,\left\{c_{1}, \ldots, c_{j}\right\} \subseteq \operatorname{Const}\left(\Gamma_{j}\right)$. Since $\exists x F(x)$ occurs infinitely many times within $H_{1}, H_{2}, H_{3}, \ldots$, then $\exists x F(x)=H_{k}$ for some $k>j$. Therefore the (b) step of our construction is applied to $\exists x F(x)$ and hence $(*)$ is proved. It follows that $\Gamma$ is $\mathcal{L}_{w}^{Q}$-rich.

The set $\Gamma$ can be extended to some $v$ which is $L_{w}^{Q}$-consistent and $L_{w}^{Q}$ - maximal. Let $\mathcal{L}_{v}=\mathcal{L}_{w}^{Q}$. The extension $v$ does not compromise richness. Indeed, if an existential formula of $\mathcal{L}_{w}^{Q}$ belongs to $v$, by $(*)$ it is also in $\Gamma$, and hence some exemplification of it is also in 
$\Gamma$ and since $\Gamma \subseteq v$, it is also in $v$. Therefore $v$ is $L_{v}^{=}$-saturated and it hence belongs to the canonical base set. Therefore:

1. $v \in W$.

2. Since $A \in \Gamma_{0}$ and $v \supseteq \bigcup_{n \in \mathcal{N}}\left(\Gamma_{n}\right)$, it follows that $A \in v$.

3. Moreover it holds true that if $\square B \in w$, then $B \in v$.

4. Since $\operatorname{Const}\left(\mathcal{L}_{w}\right) \subseteq \operatorname{Const}\left(\mathcal{L}_{w}^{Q}\right)$ it holds that $\operatorname{Const}\left(\mathcal{L}_{w}\right) \subseteq \operatorname{Const}\left(\mathcal{L}_{v}\right)$.

5. The set $v$ is $L_{w}^{Q}$-maximal: the only constants occurring in $v$ are those already present in $\Gamma$. Since by construction $\{(a=b) \mid(a=b) \in w\} \subseteq \Gamma$, it follows that for any $b$, $[b]_{w} \subseteq[b]_{v}$. On the other hand, suppose that there is some $c$ from $\operatorname{Const}\left(\mathcal{L}_{v}\right)$ which does not occur in $\operatorname{Const}\left(\mathcal{L}_{w}\right)$. Then $c$ has been added at some point in the construction of the $\Gamma_{j}$ sets. The step (3.b.1.2) is the only possible way to add the new constant $c$ to $\Gamma_{j+1}$ and it guarantees that for any $d \in \operatorname{Const}\left(\mathcal{L}\left(\Gamma_{j}\right)\right), c \neq d \in \Gamma_{j+1}$, so $c \notin[b]_{v}$ for any $b \in \operatorname{Const}\left(\mathcal{L}_{w}\right)$.

Lemma 6.9 (Truth Lemma) Given a canonical model $\mathcal{M}^{\mathrm{L}}=\langle W, \mathcal{R}, D, U, I\rangle$ for a quantified $\mathbf{N}$-Monotonic modal logic $\mathrm{L}$ extending $\mathrm{Q}_{=}^{\circ} . \mathrm{MN}$, for any formula $A \in F m a(\mathcal{L})$, for any world $w \in W$, the following holds: $\vDash_{w}^{\sigma} A\left(x_{i}\right) \Leftrightarrow A\left(\sigma\left(x_{i}\right) / x_{i}\right) \in w$.

Proof. By induction on the length of a formula $A$. We omit details of the induction base.

Suppose the length of $A$ is $n+1$ and $A$ has the form $\exists x B\left(x, y_{1}, \ldots, y_{m}\right)$.

(i) $\vDash_{w}^{\sigma} \exists x B\left(x, y_{1}, \ldots, y_{m}\right)$ iff for some $x$-variant $\tau$ of $\sigma$ such that $\tau(x) \in D_{w}, \vDash_{w}^{\tau}$ $B\left(x, y_{1}, \ldots, y_{m}\right)$. Suppose $\tau(x)=c$. Since by assumption all constants are rigid designators, by Lemma 4.7 it holds that $\vDash_{w}^{\sigma} B\left(c / x, y_{1}, \ldots, y_{m}\right)$. Hence $B\left(c / x, \sigma\left(y_{1}\right), \ldots, \sigma\left(y_{m}\right)\right) \in w$ by IH. Since $w$ is $D_{w}$-universal, $w$ is also $D_{w}$-existential by Lemma 6.4 and hence $\exists x B\left(x, \sigma\left(y_{1}\right), \ldots, \sigma\left(y_{m}\right)\right) \in w$. (ii) Assume $\exists x B\left(x, \sigma\left(y_{1}\right), \ldots, \sigma\left(y_{m}\right)\right) \in w$. Since $w$ is $D_{w^{-}}$ rich, $B\left(c / x, \sigma\left(y_{1}\right), \ldots, \sigma\left(y_{m}\right)\right) \in w$ for some $c \in D_{w}$. Thus, by $\mathrm{IH}, \vDash_{w}^{\sigma} B\left(c / x, y_{1}, \ldots, y_{m}\right)$ and, by Lemma 4.7 it holds that $\vDash_{w}^{\tau} B\left(x, y_{1}, \ldots, y_{m}\right)$ for some $w$-assignment $\tau$ which is an $x$-variant of $\sigma$ such that $\tau(x)=I_{w}(c)=c$. Therefore $\vDash_{w}^{\sigma} \exists x B\left(x, y_{1}, \ldots, y_{m}\right)$.

Assume that $\diamond A \in w$. Since the schema $\diamond A \rightarrow \diamond T$ is a theorem ${ }^{6}$ of $\mathrm{Q}_{=}^{\circ}$.MN, it follows that $\diamond T \in w$ and by Lemma 6.13 in (Calardo and Rotolo, 2014), $w$ is locally serial, i.e., for any formula $B \in F m a(\mathcal{L})$ there is a state $z$ such that $w R_{B} z$. It remains to show that for any formula $B \in F m a(\mathcal{L}), R_{B}(w) \cap|A|_{Q_{=}^{\circ} \cdot \mathrm{MN}} \neq \varnothing$, i.e., for any $B$ there is some $z$ such that $z \in R_{B}(w)$ and $A \in z$. (i) If $A$ is a theorem, then $|A|_{\mathrm{Q}_{-}^{\circ} . \mathrm{MN}}=W$ ( $A$ belongs to any maximal consistent set in $W$ ). (ii) $A$ cannot be a contradiction, otherwise $\vdash_{Q_{=}^{\circ}}$.MN $\neg A$ by $\mathbf{E F Q}, \vdash \mathrm{Q}^{\circ} . \mathrm{MN} \square \neg A, \square \neg A \in w, \neg \square \neg A \notin w$, i.e., $\diamond A \notin w$ which leads to a contradiction. (iii) If $A$ is neither a theorem nor a contradiction, both $\{A\}$ and $\{\neg A\}$ are $\mathrm{Q}_{=}^{\circ}$.MN-consistent and both $|A|_{Q_{=}^{\circ} . \mathrm{MN}}$ and $|\neg A|_{Q_{\equiv}^{\circ} . \mathrm{MN}}$ are not empty. Assume by reductio that for some $C \in F m a(\mathcal{L})$ $R_{C}$ onst $(w) \subseteq|\neg A|_{Q_{\Xi}^{\circ} . \mathrm{MN}}$. This implies that $\square C \in w$ (otherwise we would have $R_{C}(w)=W$ which is inconsistent with our assumption). Hence we have that in all $\mathrm{Q}_{=}^{\circ}$.MN-maximal sets $z, C \rightarrow \neg A \in z$ and therefore it is a theorem of $\mathrm{Q}_{=}^{\circ} . \mathrm{MN}$. Thus $\vdash \mathrm{Q}_{\underline{\circ}}^{\circ} \cdot \mathrm{MN} C \rightarrow \neg A, \vdash \mathrm{Q}_{=}^{\circ} \cdot \mathrm{MN} \square C \rightarrow$ $\square \neg A, \square \neg A \in w$ and hence $\diamond A \notin w$ which is a contradiction.

Lemma 6.10 Let $\mathcal{M}^{\mathrm{L}}:=\langle W, \mathcal{R}, D, U, I\rangle$ be a canonical model for a logic $\mathrm{L} \supseteq \mathrm{Q}_{=}^{\circ}$. MN. If $\Delta$ is an $\mathrm{L}$-consistent set of formulae, then for some $w \in W$ and some $w$-assignment $\sigma, \mathcal{M}^{\mathrm{L}} \vDash_{w}^{\sigma} D$ for any $D \in \Delta$. (cf. Corsi, 2002, Lemma 1.19)

Let $\mathrm{L}$ be any logic $L \supseteq \mathrm{Q}_{=}^{\circ}$.MN. Consider any formula $A$ such that $\nvdash_{\mathrm{L}} A$. Then $\{\neg A\}$ is $\mathrm{L}$-consistent. By Lemma 6.10 there is a world $w$ of a canonical model $\mathcal{M}^{\mathrm{L}}$ for $\mathrm{L}$ and a $w$-assignment $\sigma$ such that $\mathcal{M}^{\mathrm{L}} \neq_{w}^{\sigma} A$ and hence $\mathcal{M}^{\mathrm{L}} \neq A$.

Corollary 6.11 (Completeness of $\mathrm{Q}_{=}^{\circ} . \mathrm{MN}$ ) The logic $\mathrm{Q}_{=}^{\circ} . \mathrm{MN}$ is strongly complete with respect to the class of all multi-relational frames.

\footnotetext{
${ }^{6}$ Indeed $\vdash \mathrm{Q}_{\underline{\varrho}}^{\circ} \cdot \mathrm{MN} \perp \rightarrow \neg A$ ex falso quodlibet, $\vdash_{\mathrm{Q}_{\underline{\varrho}}^{\circ} \cdot \mathrm{MN}} \square \perp \rightarrow \square \neg A$ by $\mathbf{R M}, \vdash_{\mathrm{Q}_{\underline{e}}^{\circ} . \mathrm{MN}} \diamond A \rightarrow \diamond \mathrm{T}$ by contraposition.
} 


\subsection{Completeness and Increasing Domains}

The class of frames with restricted increasing inner domains is quite interesting on many respects. As shown in Section 5 it is closely connected to the CBF schema. In Kripke semantics the class of frames with increasing inner domains is captured by the logic $Q_{=}^{\circ} . K \oplus$ $\mathbf{C B F}$, thus showing that $\mathbf{C B F}$ is sufficient to achieve the result. The case of multi-relational frames with restricted inner domains is, however, more complex. In this section we focus on finding an axiomatisation to capture the semantic concept of restricted increasing inner domains.

It turns out that merely adding $\mathbf{C B F}$ to our system is not enough to axiomatise the logic semantically generated by the class of multi-relational frames with restricted increasing inner domains: Something more is needed. In particular, as it will become clear in the following theorem, a weak version of schema $\mathbf{C}$ is needed, namely

$$
\square \exists:=\square A \wedge \exists x_{1} \ldots \exists x_{n}\left(x_{1}=t_{1} \wedge \ldots \wedge x_{n}=t_{n}\right) \rightarrow \square\left(A \wedge \exists x_{1} \ldots \exists x_{n}\left(x_{1}=t_{1} \wedge \ldots \wedge x_{n}=t_{n}\right)\right)
$$

Let us define $\mathbf{Q}_{=}^{\circ} \cdot \mathbf{M N} \oplus \mathbf{C B F} \oplus \square \exists$ as the system obtained by adding $\mathbf{C B F}$ and $\square \exists$ to $\mathbf{Q}_{=}^{\circ} . \mathrm{MN}$. Notice that although we added a weak version of $\mathbf{C}$ (or, in other words, a stronger version of NI), this system is still non-normal. Moreover the schema $\square \exists$ does not hold in general, although it is sound in the class of multi-relational frames with finite restricted increasing inner domains.

Lemma 6.12 The schema $\square \exists$ is valid in the class of multi-relational frames with restricted increasing inner domains (as described in Lemma 5.11).

Proof. Suppose by reductio ad absurdum that there are a model $\mathcal{M}:=\langle W, \mathcal{R}, D, U, I\rangle$ based on a frame with restricted increasing inner domains and a world $w$ such that (a) $\vDash_{w}^{\sigma} \square A$, (b) $\vDash_{w}^{\sigma} \exists x_{1} \ldots \exists x_{n}\left(x_{1}=a_{1} \wedge \ldots \wedge x_{n}=a_{n}\right)$, where $\left\{a_{1}, \ldots, a_{n}\right\} \subseteq D_{w}$, and (c) $\vDash_{w}^{\sigma} \diamond\left(\neg A \vee \forall x_{1} \ldots \forall x_{n}\left(x_{1} \neq a_{1} \vee \ldots \vee x_{n} \neq a_{n}\right)\right)$. Clearly by (c) $w$ is locally serial and by (a) there is a relation $R_{i}$ such that $R_{i}(w) \subseteq\|A\|_{I}^{\sigma}$. By definition there is a relation $R_{k}$ such that $R_{k}(w) \subseteq R_{i}(w)$ and the inner domains are increasing. Since $R_{k}(w) \subseteq\|A\|_{I}^{\sigma}$, by (c) there must be a world $v \in R_{k}(w)$ such that $\vDash_{v}^{\sigma} \forall x_{1} \ldots \forall x_{n}\left(x_{1} \neq a_{1} \vee \ldots \vee x_{n} \neq a_{n}\right)$. Since any $a_{i}$ belongs to $D_{w}$, the inner domains are not increasing, thus reaching a contradiction.

Theorem 6.13 (Completeness of $\mathrm{Q}_{=}^{\circ} . \mathrm{MN} \oplus \mathbf{C B F} \oplus \square \exists$ ) The logic $\mathrm{Q}_{=}^{\circ} . \mathrm{MN} \oplus \mathbf{C B F} \oplus \square \exists$ is complete with respect to the class of multi-relational frames with finite restricted increasing inner domains, i.e. frames with the following property: $\forall w \in W$, if $w$ is locally serial, then for all $R_{j}$, there is some relation $R_{i}$ such that $R_{i}(w) \subseteq R_{j}(w)$ and for all worlds $v$ in $R_{i}(w)$, $D_{w} \subseteq D_{v}$, as in Lemma 5.11 .

Proof. Let $\mathcal{M}$ be a canonical model for $\mathrm{Q}_{=}^{\circ} \cdot \mathrm{MN} \oplus \mathbf{C B F}$. Take any world $w$ and assume it is locally serial. Then the schema $\diamond T$ belongs to $w$. By Lemma 6.8 it follows that for any formula $B_{i} \in \operatorname{Fma}(\mathcal{L})$ there is a world $v_{i}$ such that $w R_{B_{i}} v_{i}$ and $\operatorname{Const}\left(\mathcal{L}_{w}\right) \subseteq \operatorname{Const}\left(\mathcal{L}_{v_{i}}\right)$ and for any individual constant $c \in \operatorname{Const}\left(\mathcal{L}_{w}\right),[c]_{w}=[c]_{v_{i}}$. This implies that $U_{w} \subseteq U_{v_{i}}$. Take any $[c] \in D_{w}$; by definition it holds that $\exists x(x=c) \in w$. From these facts it follows that $\vDash_{w}^{\sigma} x=c$ for some $\sigma$ such that $\sigma(x) \in D_{w}$ and, moreover, for each $i, \vDash_{v_{i}}^{\sigma} x=c$. Hence $\vDash_{w}^{\sigma} \diamond(x=c)$ and since $\sigma(x) \in D_{w}, \exists x \diamond(x=c) \in w$. By CBF, $\diamond \exists x(x=c) \in w$. This means that for every existing individual $b$ from $D_{w}$, for any relation $R_{B}$, there is a world $t \in R_{B}(w)$ such that $b \in D_{t}$.

Now we want to show that the property expressed by Lemma 5.11 holds. Take any relation $R_{A}$. By definition of canonical model, the set $R_{A}(w)$ is such that either $(1) R_{A}(w)=$ $\|A\|_{I}$, or (2) $R_{A}(w)=\|\top\|_{I}$. If (1), then $\square A \in w$, hence the formula $\square A \wedge \wedge_{a \in D_{w}} \exists x(x=a) \in w$. By axiom $\square \exists:=\square A \wedge \exists x_{1}\left(x_{1}=t_{1}\right) \wedge \ldots \wedge \exists x_{n}\left(x_{n}=t_{n}\right) \rightarrow \square\left(A \wedge \exists x_{1}\left(x_{1}=t_{1}\right) \wedge \ldots \wedge \exists x_{n}\left(x_{n}=\right.\right.$ $\left.\left.t_{n}\right)\right)$, we get $\square\left(A \wedge \wedge_{a \in D_{w}} \exists x(x=a)\right) \in w$. Let us denote this formula by the letter $C$. Clearly, $R_{C}(w) \subseteq R_{A}(w)$ and for any world $v \in R_{C}(w)$, it holds that $D_{w} \subseteq D_{v}$. Likewise, if (2) holds, $\square T \in w$ and by a similar argument we can show that the domains are increasing. 


\section{The Case of Barcan Formula}

\subsection{BF and CGF: Semantic Considerations}

If compared to $\mathbf{C B F}$, additional technical difficulties make $\mathbf{B F}$ harder to handle. The remainder of this section provides some preliminary but interesting results.

Similar to the case with $\mathbf{C B F}$, the invalidity of the BF schema follows immediately from its analogous result in Kripke semantics.

Lemma 7.1 BF is not valid in the class of multi-relational frames.

Proof. Consider the following model: $\mathcal{M}:=\left\langle W, R_{1}, D, U, I\right\rangle$ where $W:=\{w, z\}, R_{1}:=$ $\{\langle w, z\rangle\}, D_{w}=\{a\}, D_{z}=\{b\}, U_{w}=U_{z}=\{a, b\}, P(a) \in I_{z}(P)$ and $P(b) \notin I_{z}(P)$. Then it holds that for any $x$-variant of a $\sigma$-assignment $\tau$ such that $\tau(x) \in D_{w}, \vDash_{w}^{\tau} \square P(x)$ and hence $\vDash_{w}^{\sigma} \forall x \square P(x)$. Moreover there is an $x$-variant $\theta$ of $\sigma$ such that $\theta(x) \in D_{z}$ and $P(x) \in I_{z}^{\theta}(P)$, i.e. $\theta(x)=b$ and since $w R_{1} z$ it holds that $\vDash_{w}^{\sigma} \diamond \exists x \neg P(x)$. (See Figure 3)

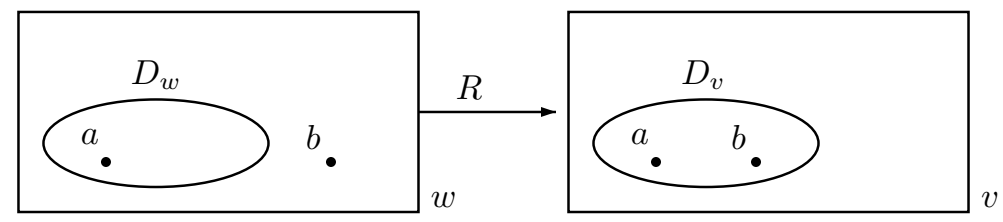

Figure 3: A Kripke frame to build a countermodel for $B F$.

Lemma 7.2 (BF Characterisation Result) For any multi-relational frame $\mathcal{F}$ := $\langle W, \mathcal{R}, D, U\rangle, \mathcal{F} \vDash \forall x \square A \rightarrow \square \forall x A$ iff $\forall w \in W$, either there is an $R_{i} \in \mathcal{R}$ such that $R_{i}(w)=\varnothing$ or for any (possibly infinite) sequence of worlds $z_{1}, z_{2}, \ldots$ of length $n$ (if the sequence is infinite $n$ is $\aleph_{0}$ ) such that $n$ is also the cardinality of $\mathcal{R}$ and $w R_{k} z_{k}$ for any $R_{k} \in \mathcal{R}$, for any (possibly infinite) sequence $R_{j_{1}}, R_{j_{2}}, \ldots$ of length $m$ where $\left\|D_{w}\right\|=m$, i.e., $m$ is the number of individuals belonging to the inner domain of $w$, there is a world $t \in\left\{z_{1}, z_{2}, \ldots\right\} \cap \bigcap_{i \leq m} R_{j_{i}}(w)$ such that $D_{w} \supseteq D_{t}$.

Proof. $(\Rightarrow)$ Assume that there is a $w \in W$ such that for all $R_{i} \in \mathcal{R}, R_{i}(w) \neq \varnothing$ and for some (possibly infinite) sequence of worlds $z_{1}, z_{2}, \ldots$ such that $w R_{k} z_{k}$ for any $R_{k} \in \mathcal{R}$, for some (possibly infinite) sequence of relations $R_{j_{1}}, R_{j_{2}}, \ldots$ the following holds: for all $t \in W$, if $t \in\left\{z_{1}, z_{2}, \ldots\right\} \cap R_{j_{1}}(w) \cap \ldots \cap R_{j_{m}}(w)$ then $D_{w} \subset D_{t}$. We shall now define an interpretation $I$ in order to build a countermodel for BF in this frame. Let $D_{w}:=\left\{d_{1}, d_{2}, \ldots\right\}$ of cardinality $m$ (not necessarily finite), and consider the $w$-assignments $\sigma_{1}, \sigma_{2}, \ldots$ such that for any $\sigma_{i}$, $\sigma_{i}(x)=d_{i}$. For some unary predicate $P$, for any $i$, let $\|P(x)\|_{I}^{\sigma_{i}}:=R_{j_{i}}(w)$. Then clearly $\vDash_{w}^{\sigma_{i}} \square P(x)$ for each $i$ and hence, since $\sigma_{1}, \sigma_{2}, \ldots$ are all the $x$-variant of a $w$-assignment $\sigma$ such that $\sigma_{i} \in D_{w}$ for each $i, \vDash_{w}^{\sigma} \forall x \square P(x)$. Consider now any $z_{i}$ from the sequence $z_{1}, z_{2}, \ldots$ and define a $z_{i}$-assignment $\vartheta_{i}$ such that $\vartheta_{i}(x) \notin I_{z_{i}}^{\vartheta_{i}}(P(x))$ and $\vartheta_{i}(x) \in D_{z_{i}}$. Notice that it is always possible to define such an assignment. In fact even if $z_{i} \in R_{j_{1}}(w) \cap \ldots \cap R_{j_{m}}(w)$, by assumption we have that $D_{z_{i}} \supset D_{w}$. Hence $\vDash_{z_{i}}^{\vartheta_{i}} \neg P(x)$ and hence $\vDash_{z_{i}}^{\sigma} \neg \exists x P(x)$ which implies that $\not_{w}^{\sigma} \square \forall x P(x)$.

$(\Leftarrow)$ Assume that for some frame $\mathcal{F}, \mathcal{F} \not \forall x \square A \rightarrow \square \forall x A$ for some formula $A$. Then there are a world $w$, an interpretation $I$ and an assignment $\sigma$ such that $\left(\right.$ a) $\vDash_{w}^{\sigma} \forall x \square A(x)$ and (b) $\vDash_{w}^{\sigma} \diamond \exists x \neg A(x)$. Given that $D_{w}:=\left\{d_{1}, d_{2}, \ldots\right\}$ and $\left\|D_{w}\right\|=m$, from (a) it follows that there are $m w$-assignments $\sigma_{1}, \sigma_{2}, \ldots$ which are all the $x$-variants of $\sigma$ such that $\sigma_{i}(x) \in D_{w}$ and $\vDash_{w}^{\sigma_{i}} \square A(x)$. Thus there is a set of relations $\left\{R_{1}, R_{2}, \ldots\right\}$ of cardinality $m$ such that for any $R_{i}, R_{i}(w) \subseteq\|A(x)\|_{I}^{\sigma_{i}}$.

From (b) it follows that there is a sequence $z_{1}, z_{2}, \ldots$ of worlds such that for each $R_{i}$, $w R_{i} z_{i}$ (hence for each $R_{i}, R_{i}(w) \neq \varnothing$ ) and for each $i, \vDash_{z_{i}}^{\sigma} \exists x \neg A(x)$. Thus for each $z_{i}$ there is some $z_{i}$-assignment $\vartheta_{i}$ such that $\vartheta_{i}(x) \in D_{z_{i}}$ and $\vDash_{z_{i}}^{\vartheta_{i}} \exists x \neg A(x)$. 
Clearly all the worlds $t$ belonging to the intersection $\bigcap_{i \leq m} R_{j_{i}}(w)$ are such that $\vDash_{t}^{\sigma_{i}} A(x)$ for all the $x$-variants of $\sigma$ such that $\sigma_{i}(x) \in D_{w}$ and hence if a world $z_{i}$ belongs to such intersection, the $z_{i}$-assignment $\vartheta_{i}$ must be such that $\vartheta_{i}(x) \in D_{z_{i}}$ but $\vartheta_{i}(x) \notin D_{w}$ and therefore for any such world $D_{w} \subset D_{z_{i}}$.

Again, mirroring Kripke semantics, the schemata BF and CGF := $\square x A \rightarrow \exists x \square A$ are equivalent.

Lemma 7.3 The schemata $\mathbf{B F}$ and $\mathbf{C G F}$ characterise the same class of multi-relational frames.

Proof. $(\Rightarrow)$ Assume that $\exists w \in W$ such that for all $R_{i} \in \mathcal{R}, R_{i}(w) \neq \varnothing$ and for some (possibly infinite) sequence $z_{1}, z_{2}, \ldots$ such that $w R_{k} z_{k}$ for any $R_{k} \in \mathcal{R}$, for some (possibly infinite) sequence of relations $R_{j_{1}}, R_{j_{2}}, \ldots$ of length $m$ (if the sequence is infinite $m$ is $\aleph_{0}$ ), where $m$ is the number of individuals belonging to $D_{w}$, the following holds: for all $t \in W$, if $t \in\left\{z_{1}, z_{2}, \ldots\right\} \bigcap_{i \leq m} R_{j_{i}}(w)$ then $D_{w} \subset D_{t}$.

We shall now define an interpretation $I$ in order to build a countermodel for CGF in this frame. Let $D_{w}:=\left\{d_{1}, d_{2}, \ldots\right\}$ of cardinality $m$ and consider the sequence of $w$-assignments $\sigma_{1}, \sigma_{2}, \ldots$ of length $m$ such that for any $\sigma_{i}, \sigma_{i}(x)=d_{i}$. For some unary predicate $P$, for any $i$, let $\|P(x)\|_{I}^{\sigma_{i}}:=R_{j_{i}}(w)$. Then clearly $\vDash_{w}^{\sigma_{i}} \square P(x)$ for each $i$ and hence, since $\sigma_{1}, \sigma_{2}, \ldots$ are all the $x$-variants of a $w$-assignment $\sigma$ such that $\sigma_{i} \in D_{w}$ for each $i, \vDash_{w}^{\sigma} \forall x \square P(x)$.

Consider now any $z_{i}$ from the sequence $z_{1}, z_{2}, \ldots$ and define a $z_{i}$-assignment $\vartheta_{i}$ such that $\vartheta_{i}(x) \notin I_{z_{i}}^{\vartheta_{i}}(P(x))$ and $\vartheta_{i}(x) \in D_{z_{i}}$. Notice that it is always possible to define such an assignment. In fact even if $z_{i} \in \bigcap_{i \leq m} R_{j_{i}}(w)$, by assumption we have that $D_{z_{i}} \supset D_{w}$. Hence $\vDash_{z_{i}}^{\vartheta_{i}} \neg P(x)$ and hence $\vDash_{z_{i}}^{\sigma} \neg \exists x P(x)$ which implies that $\vDash_{w}^{\sigma} \square \forall x P(x)$.

$(\Leftarrow)$ Assume that for some frame $\mathcal{F}, \mathcal{F} \neq \forall x \square A \rightarrow \square \forall x A$ for some formula $A$. Then there are a world $w$, an interpretation $I$ and an assignment $\sigma$ such that $(\mathrm{a}) \vDash_{w}^{\sigma} \forall x \square A(x)$ and (b) $\vDash_{w}^{\sigma} \diamond \exists x \neg A(x)$. Given that $D_{w}:=\left\{d_{1}, \ldots, d_{m}\right\}$, from (a) it follows that there are $\sigma_{1}, \sigma_{2}, \ldots$ $w$-assignments which are all the $x$-variants of $\sigma$ such that $\sigma_{i}(x) \in D_{w}$ and $\vDash_{w}^{\sigma_{i}} \square A(x)$. Thus there is a set of relations $R_{1}, R_{2}, \ldots$ such that for any $R_{i}, R_{i}(w) \subseteq\|A(x)\|_{I}^{\sigma_{i}}$.

From (b) it follows that there is a sequence $z_{1}, z_{2}, \ldots$ of length $n$ of worlds such that for each $R_{i}, w R_{i} z_{i}$ (hence for each $R_{i}, R_{i}(w) \neq \varnothing$ ) and for each $i, \vDash_{z_{i}}^{\sigma} \exists x \neg A(x)$. Thus for each $z_{i}$ there is some $z_{i}$-assignment $\vartheta_{i}$ such that $\vartheta_{i}(x) \in D_{z_{i}}$ and $\vDash_{z_{i}}^{\vartheta_{i}} \exists x \neg A(x)$.

Clearly all the worlds $t$ belonging to the intersection $\bigcap_{i \leq m} R_{j_{i}}(w)$ are such that $\vDash_{t}^{\sigma_{i}} A(x)$ for all the $x$-variants of $\sigma$ such that $\sigma_{i}(x) \in D_{w}$ and hence if a world $z_{i}$ belongs to such intersection, the $z_{i}$-assignment $\vartheta_{i}$ must be such that $\vartheta_{i}(x) \in D_{z_{i}}$ but $\vartheta_{i}(x) \notin D_{w}$ and therefore for any such world $D_{w} \subset D_{z_{i}}$.

By translating in terms of multi-relational semantics also Stolpe (2003)'s CUPI condition (see Theorem 2.8 it is quite straightforward to prove a weaker correspondence result for $\mathbf{B F}$ in multi-relational models. However this implies conditions on models instead of frames.

Lemma 7.4 (CUPI models) For any multi-relational model $\mathcal{M}:=\langle W, \mathcal{R}, D, U, I\rangle, \mathcal{M} \vDash$ $\forall x \square A \rightarrow \square \forall x A$ if and only if for any world $w$ the following holds: given a w-assignment $\sigma$, if for any $x$-variant $\sigma_{i}$ such that $\sigma_{i}(x) \in D_{w}$ there exists a relation $R_{i}$ such that $R_{i}(w) \subseteq$ $\|A(x)\|_{I}^{\sigma_{i}}$, then there is a relation $R_{k}$ such that $R_{k}(w) \subseteq\|\forall x A\|_{I}^{\sigma}$.

Proof. The left to right arrow is trivial. For the other direction consider the contrapositive proposition. Assume that for some world $w \in W$ it holds true that, for all $w$-assignments $\sigma$, (a) for any $x$-variant $\sigma_{i}$ of $\sigma, \sigma_{i}(x) \in D_{w}$, there exists a relation $R_{i}$ such that $R_{i}(w) \subseteq$ $\|A(x)\|_{I}^{\sigma_{i}}$ and (b) for any relation $R_{k}, R_{k}(w) \nsubseteq\|\forall x A\|_{I}^{\sigma}$. From (a) it follows that for any $\sigma_{i}, \vDash_{w}^{\sigma_{i}} \square A(x)$ and hence $\vDash_{w}^{\sigma} \forall x \square A(x)$ whereas from (b) it follows that $\nvdash_{w}^{\sigma} \square \forall x A$, thus $\sharp_{w}^{\sigma} \forall x \square A \rightarrow \square \forall x A$. 


\subsection{The Independence of the Schemata C and BF}

Recall that the schema $\mathbf{C}$ imposes on frames the following property: $\mathcal{F} \vDash \square A \wedge \square B \rightarrow \square(A \wedge B)$ iff for any world $w$, for any relation $R_{i}, R_{k}$ there exists a relation $R_{j}$ such that $R_{j}(w) \subseteq$ $R_{k}(w) \cap R_{i}(w)$. Hence for any $\mathbf{C}$-frame, the characterisation result for $\mathbf{B F}$ is the following:

Lemma 7.5 (BF on C-frames) Let $\mathcal{F}$ be any $\mathbf{C}$-frame. Then for any world $w \in W$, given that $\left\|D_{w}\right\|=k$, if $w$ is locally serial, then for all sets of relations with cardinality up to $k$ $\left\{R_{1}^{i}, \ldots, R_{k}^{i}\right\}$, for all worlds $v \in R_{1}^{i} \cap \ldots \cap R_{k}^{i}$ it holds that $D_{w} \supseteq D_{v}$.

Proof. Indeed if we assume that the BF-condition does not hold on a $\mathbf{C}$-frame, we would get the following situation. For some world $w$ such that $w$ is locally serial and $\left\|D_{w}\right\|=k$, there is some set $R_{1}, \ldots, R_{k}$ of relations such that for some world $v \in R_{1}(w) \cap \ldots \cap R_{k}(w)$ it holds that $D_{w} \nsupseteq D_{v}$. It is easy to build a counter-model in such situation. In Kripke semantics this is equivalent to denying decreasing domains.

It is easy to find a frame validating $\mathbf{C}$ but not $\mathbf{B F}$ : It is enough to consider the frame described in Lemma 7.1, which is Kripkean and hence validates $\mathbf{C}$.

It is not hard to show that the schema BF does not imply C. Below we describe a countermodel for $\mathbf{C}$ based on a frame for BF. Consider the model $\mathcal{M}:=\langle W, \mathcal{R}, D, U, I\rangle$ where $W=\left\{w, z_{1}, z_{2}\right\}, \mathcal{R}:=\left\{R_{1}, R_{2}\right\}, R_{1}:=\left\{\left\langle w, z_{1}\right\rangle\right\}, R_{2}:=\left\{\left\langle w, z_{2}\right\rangle\right\}, D_{w}=D_{z_{1}}=D_{z_{2}}=\{d\}$, $\sigma$ is a $w$-assignment such that $\sigma(x)=d$. Let $P$ and $Q$ be two unary predicates such that $\|P(x)\|_{I}^{\sigma}=\left\{z_{1}\right\}$ and $\|Q(x)\|_{I}^{\sigma}=\left\{z_{2}\right\}$. Then $\vDash_{w}^{\sigma} \square P(x) \wedge \square Q(x)$ but $\neq_{w}^{\sigma} \square(P(x) \wedge Q(x))$. Moreover $\mathcal{M}$ is built on a frame which fulfills the conditions imposed by BF. Indeed $w$ is locally serial but there is only one tuple $\left\langle z_{1}, z_{2}\right\rangle$ such that $w R_{1} z_{1}$ and $w R_{2} z_{2}$. The number of individuals from $D_{w}$ is 1 , thus: $z_{1} \in\left\{z_{1}, z_{2}\right\} \cap R_{1}(w)$ and $D_{w} \subseteq D_{z_{1}}$ and $z_{2} \in\left\{z_{1}, z_{2}\right\} \cap R_{2}(w)$ and $D_{w} \subseteq D_{z_{2}}$. Notice that any countermodel for $\mathbf{C}$ based on a $\mathbf{B F}$-frame must contain a locally serial world $w$ whose inner domain contains at most one individual and the frame of such model should have more than one relation.

Corollary 7.6 The schemata $\mathbf{B F}$ and $\mathbf{C}$ are semantically mutually independent.

The importance of this result is limited at the moment, as it lays only on semantic grounds. In fact, without a completeness result, one cannot infer that adding BF to a system does not generate normal systems, i.e., it is not yet clear whether BF does or does not syntactically imply $\mathbf{C}$.

\section{The Role of Identity}

The choice of a language with the identity relation can be driven by different reasons. First of all, the expressive power increases greatly. Nevertheless, there is also a technical reason behind it. Working with the identity symbol allowed us to build canonical models for CBFframes, a very difficult goal to be achieved without it. It is actually much easier to prove completeness for systems without identity, at least for those systems which do not include any form of the Barcan schemata.

\subsection{Completeness without Identity}

Below we present a completeness theorem for an analogous system built on a language without identity. The axiomatic system $Q^{\circ} . M N$ is obtained by deleting all the schemata concerning identity:

The system $\mathrm{Q}^{\circ}$.MN contains the following axioms and inference rules:

- Propositional tautologies;

- $\mathbf{U I}^{\circ}:=\forall y(\forall x A(x) \rightarrow A(y / x))$

- $\forall x \forall y A \leftrightarrow \forall y \forall x A$

- $A \rightarrow \forall x A, x$ not free in $A$

- $\forall x(A \rightarrow B) \rightarrow(\forall x A \rightarrow \forall x B)$
- $\mathbf{M}:=\square(A \wedge B) \rightarrow(\square A \wedge \square B)$

- $\mathbf{N}:=\square T$ to $\mathbf{Q}_{=}^{\circ} . \mathrm{E}$

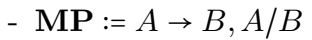

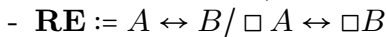

- $\mathbf{U G}:=A / \forall x A$ 
Theorem 8.1 (Soundness) The system $\mathrm{Q}^{\circ} . \mathrm{MN}$ is sound with respect to the class of all multi-relational frames with varying inner domains.

Proof. It follows from Theorem 6.1

Lemma 8.2 Let $\Delta$ be an L-consistent set of sentences of $\mathcal{L}$. Then for some not-empty denumerable set $C$ of new constants, there is a set $\Pi$ of sentences of $\mathcal{L}^{C}$ such that $\Delta \subseteq \Pi$, $\Pi$ is $L^{C}$-maximal, $\Pi$ is $Q$-universal and $Q$-rich for some set $Q \subseteq \operatorname{Const}\left(\mathcal{L}^{C}\right)$. (cf. (Corsi), 2002, Lemma 1.16))

Definition 8.3 (Non-normal Canonical Models) Let $\mathrm{L}$ be a non-normal quantified modal logic on the language $\mathcal{L}$ such that $L \supseteq \mathrm{Q}^{\circ}$.MN. Let $Q$ be a set of constants of cardinality $\aleph_{0}$ such that $Q \supset \operatorname{Const}(\mathcal{L})$ and $\|Q-\operatorname{Const}(\mathcal{L})\|=\aleph_{0}$. A non-normal canonical model $\mathcal{M}^{\mathrm{L}}=\langle W, \mathcal{R}, D, U, I\rangle$ for $\mathrm{L}$ is defined as follows:

- $W$ is the class of all $L_{w}$-saturated sets of sentences $w$, where $\mathcal{L}_{w}=\mathcal{L}^{S}$ for some set $S$ of constants such that $\operatorname{Const}\left(\mathcal{L}^{S}\right) \neq \varnothing, S \subset Q$ and $\left\|Q-\operatorname{Const}\left(\mathcal{L}^{S}\right)\right\|=\aleph_{0}$.

- For any formula $A \in F m a(\mathcal{L})$ let $R_{A}$ be a binary relation over $W$. For all $w, v \in W$, $w R_{A} v$ iff $\square A \in w \Rightarrow A \in v$. The set of relations $\mathcal{R}$ is the collections of all such relations.

- $D_{w}=\left\{c \in \operatorname{Const}\left(\mathcal{L}_{w}\right) \mid \forall x A \rightarrow A(c / x) \in w\right.$, for all sentences $\forall x A$ of $\left.\mathcal{L}_{w}\right\}$

- $U_{w}=\operatorname{Const}\left(\mathcal{L}_{w}\right)$

- $I_{w}(c)=c$

- $I_{w}\left(P^{n}\right)=\left\{\left\langle c_{1}, \ldots, c_{n}\right\rangle \mid P^{n}\left(c_{1}, \ldots, c_{n}\right) \in w\right\}$.

Lemma 8.4 (Existence lemma) Given a canonical model $\mathcal{M}$ for $\mathrm{Q}^{\circ} . \mathrm{MN}$, for any $w \in W$, if $\diamond A \in w$, then for any formula $B \in F m a(\mathcal{L})$ there is a state $z$ such that $w R_{B} z$ and $A \in z$.

The proof follows directly from Lemma 6.14 by Calardo and Rotolo (2014).

Lemma 8.5 (Truth Lemma) Given a canonical model $\mathcal{M}^{\mathrm{L}}=\langle W, \mathcal{R}, D, U, I\rangle$ for a quantified $\mathbf{N}$-Monotonic modal logic $\mathrm{L}$ extending $\mathrm{Q}^{\circ}$.MN, for any formula $A \in F m a(\mathcal{L})$, for any world $w \in W$, the following holds: $\vDash_{w}^{\sigma} A\left(x_{i}\right) \Leftrightarrow A\left(\sigma\left(x_{i}\right) / x_{i}\right) \in w$.

Proof. By induction on the length of a formula $A$. We omit details of the induction base. Suppose the length of $A$ is $n+1$ and $A$ has the form $\exists x B\left(x, y_{1}, \ldots, y_{m}\right)$.

(i) $\vDash_{w}^{\sigma} \exists x B\left(x, y_{1}, \ldots, y_{m}\right)$ iff for some $x$-variant $\tau$ of $\sigma$ such that $\tau(x) \in D_{w}, \vDash_{w}^{\tau}$ $B\left(x, y_{1}, \ldots, y_{m}\right)$. Suppose $\tau(x)=c$. Since by assumption all constants are rigid designators, by Lemma 4.7 it holds that $\vDash_{w}^{\sigma} B\left(c / x, y_{1}, \ldots, y_{m}\right)$. Hence $B\left(c / x, \sigma\left(y_{1}\right), \ldots, \sigma\left(y_{m}\right)\right) \in w$ by IH. Since $w$ is $D_{w}$-universal, $w$ is also $D_{w}$-existential by Lemma 6.4 and hence $\exists x B\left(x, \sigma\left(y_{1}\right), \ldots, \sigma\left(y_{m}\right)\right) \in w$.

(ii) Assume $\exists x B\left(x, \sigma\left(y_{1}\right), \ldots, \sigma\left(y_{m}\right)\right) \in w$. Since $w$ is $D_{w}$-rich, $B\left(c / x, \sigma\left(y_{1}\right), \ldots, \sigma\left(y_{m}\right)\right) \in w$ for some $c \in D_{w}$. Thus, by $\mathrm{IH}, \vDash_{w}^{\sigma} B\left(c / x, y_{1}, \ldots, y_{m}\right)$ and, by Lemma 4.7 it holds that $\vDash_{w}^{\tau} B\left(x, y_{1}, \ldots, y_{m}\right)$ for some $w$-assignment $\tau$ which is an $x$-variant of $\sigma$ such that $\tau(x)=I_{w}(c)=c$. Therefore $\vDash_{w}^{\sigma} \exists x B\left(x, y_{1}, \ldots, y_{m}\right)$. If $l g(A)=n+1$ and $A$ has the form $\square B$, please refer to Lemma 48 (Truth Lemma) in (Calardo and Rotolo 2014).

Lemma 8.6 Let $\mathcal{M}^{\mathrm{L}}:=\langle W, \mathcal{R}, D, U, I\rangle$ be a canonical model for a logic $L \supseteq \mathrm{Q}^{\circ}$.MN. If $\Delta$ is an $\mathrm{L}$-consistent set of formulae, then for some $w \in W$ and some $w$-assignment $\sigma, \mathcal{M}^{\mathrm{L}} \vDash_{w}^{\sigma} D$ for any $D \in \Delta$. (cf. Corsi, 2002, Lemma 1.19)).

Let $\mathrm{L}$ be any logic $L \supseteq \mathrm{Q}^{\circ}$.MN. Consider any formula $A$ such that $\nvdash_{L} A$. Then $\{\neg A\}$ is $\mathrm{L}$-consistent. By Lemma 6.10 there is a world $w$ of a canonical model $\mathcal{M}^{\mathrm{L}}$ for $\mathrm{L}$ and a $w$-assignment $\sigma$ such that $\mathcal{M}^{\mathrm{L}} \neq_{w}^{\sigma} A$ and hence $\mathcal{M}^{\mathrm{L}} \neq A$.

Theorem 8.7 (Completeness of $\mathrm{Q}^{\circ} . \mathrm{MN}$ ) The logic $\mathrm{Q}^{\circ} . \mathrm{MN}$ is strongly complete with respect to the class of all multi-relational frames. 


\subsection{Some Remarks on Identity}

The completeness proof for a system without identity follows from the propositional results (see Calardo and Rotolo, 2014) and from those stated by Corsi (2002). However, things change radically when the identity symbol is included. As Corsi (2002) observes:

In a language with identity, the fact that constants are rigid designators can be expressed by the schema:

$$
(x=a) \rightarrow \square(x=a)
$$

Therefore (...) all the systems of Q.M.L. with identity we are going to discuss are bound to be systems with rigid terms. (Corsi, 2002, 1499)

However this ceases to be true in the broader framework of multi-relational semantics. In fact if there is only one relation within a frame (the Kripkean case), the problem of rigidity can be easily solved by stating that if two names denote the same individual in a world, then this is bound to be the case in all accessible worlds. On the other hand, if interpreted in the multi-relational case, this would only state that under some accessibility relation this couple of names denote the same individual. This is obviously not enough. Our version of the necessity of identity schema

$$
\mathbf{N I}:=\square A \wedge(a=b) \rightarrow \square(A \wedge(a=b))
$$

says something more. It states that for any formula, i.e., semantically, for any relation, this must hold. It is a restricted form of axiom $\mathbf{C}$, which holds only for specific formulae, namely identities. The same holds for the necessity of diversity, ND. The resulting system is still a proper subset of $\mathrm{K}$ even though it includes a restricted version of the aggregation schema C.

This may explain what happened in Lemma 6.8, in the construction of the set $\Gamma_{1}$. In the proof of Lemma 6.8 , the set $\Gamma_{0}$ is built to ensure the truth of modal formulae:

$$
\Gamma_{0}:=
$$

1. If $\square B \in w$, then $\Gamma_{0}:=\{B\} \cup\{A\}$

2. otherwise, $\Gamma_{0}:=\{A\}$.

This is not sufficient to preserve rigidity of denotation. However, it can be amended by adding a further step in the construction:

$$
\Gamma_{1}:=\Gamma_{0} \cup\{(a=b) \mid(a=b) \in w\}
$$

This further step is necessary to keep rigidity of designation as well as the validity of axiom NI. Moreover, the consistency of $\Gamma_{1}$ is guaranteed by the presence of NI in the system.

This is even more obvious within the proof of Theorem 6.13. where we make an essential use of identity formulae. In fact, when it comes to prove completeness results for systems including $\mathbf{C B F}$, if identity is not present, it is very difficult to denote the same individual across different worlds. One may think about adding an existence predicate, but this would be just a definite description in disguise. The standard way to introduce an existence predicate is to add to the language a unary predicate symbol $E$ whose extension, for any world, is equal to the inner domain:

$$
\vDash_{w}^{\sigma} E(x) \text { if and only if } \sigma(x) \in D_{w}
$$

This is clearly equivalent to state that $E(a)$ is satisfied in a world if and only if the formula $\exists x(x=a)$ holds. Hence, adding an existence predicate would make very little difference, if not at all, on the technical level and would not take us any closer to proof completeness for systems including CBF. 


\section{Summary and Further Work}

This paper provided a semantic study in multi-relational semantics of quantified N-Monotonic modal logics with varying domains with and without the identity symbol $\left(Q_{=}^{\circ} . M N\right.$ and $\left.\mathrm{Q}^{\circ} . \mathrm{MN}\right)$. We also identified conditions on frames to characterize Barcan and Ghilardi schemata and presented some completeness results. The characterisation of $\mathbf{B F}$ and $\mathbf{C B F}$ in multi-relational frames with varying domains has shown that $\mathbf{B F}$ and $\mathbf{C}$ are mutually independent and that $\mathbf{M}$ does not imply the validity of $\mathbf{C B F}$ ( $\mathbf{M}$ is an axiom of $\mathbf{M N}$ ). This fact was suggested for classical modal systems by Stolpe (2003) but unfortunately that work used only models and not frames.

In detail, in this paper

- We provided a completeness result for $\mathrm{Q}_{=}^{\circ} \cdot \mathrm{MN}$;

- We provided a completeness results for $\mathrm{Q}^{\circ} . \mathrm{MN}$;

- We proved the semantic equivalence for $\mathbf{C B F}$ and $\mathbf{G F}$, and for BF and CGF;

- We provided frame characterisation results for both $\mathbf{C B F}$, and BF;

- We provided a completeness result for $\mathbf{Q}_{=}^{\circ} \cdot \mathrm{MN} \oplus \mathbf{C B F} \oplus \square \exists$;

- We showed that Schema $\mathbf{M}$ does not entail CBF;

- We proved that schema $\mathbf{C}$ and $\mathbf{B F}$ are semantically independent.

Problems related to the completeness of non-normal systems, with and without identity, that include BF, are still open and require a deeper analysis. In systems without identity, this problem concerns both $\mathbf{C B F}$ and BF. Moreover, we see other important directions for future research.

A preliminary question - which is out of scope for this paper - regards the general relation between multi-relational and neighbourhood semantics. For propositional non-normal modal logics the two semantics are equivalent (Governatori and Rotolo, 2005). However, it is not obvious if they are still equivalent (in regard, e.g., to completeness and incompleteness results) above K: Normal systems do not necessarily make Governatori and Rotolo (2005)'s semantics collapse on Kripke's. Regarding quantified modal logics, a full comparison of the two semantics is anyway needed.

An immediate extension of the present work is to consider constant domains (FOL $\oplus$ MN). It is definitely less trivial to adopt Governatori and Rotolo (2005)'s semantics and study the open problem that Arló-Costa (2011) mentioned for FOL $\oplus \mathrm{E} \oplus \mathbf{C B F}$, as well as to investigate other quantified (classical, monotonic, and regular) systems with constant and varying domains.

Finally, a question concerning (Kracht and Wolter, 1999 Gasquet and Herzig, 1996)'s proof that non-normal modal logics can be simulated by a normal modal logic with three modalities. The key idea is to translate the neighbourhood models into Kripke models with three suitable relations as well as the $\square$-formulae into three-modality formulae. This result, which holds for $\mathrm{MN}$, is of great interest in the context of multi-relational semantics, whose structures can in fact recall Kripke frames for multi-modal logics: indeed, $\square$-formulae are evaluated in multi-relational semantics by considering one existing relation among a set of accessibility relations, a plurality of relations that occurs, too, in Kripke structures for multi-modal logics. However, when predicate calculi are added to the propositional modal base we can obtain unexpected interactions between Barcan schemata and modal axioms: while for propositional multi-relational frame, one can easily adapt (Kracht and Wolter 1999. Gasquet and Herzig, 1996)'s translation, it is not clear that the translation holds for first-order extensions. Hence, extending Kracht and Wolter (1999)'s case to quantified non-normal modal logics is an open question.

\section{References}

Arló-Costa, H. (2002). First order extensions of classical systems of modal logic. Studia Logica 71, 87-118. 
Arló-Costa, H. (2011). Quantified modal logic. In A. Gupta and J. van Benthem (Eds.), Logic and Philosophy Today, vol. 2. London: College Publications.

Arló-Costa, H. L. and E. Pacuit (2006). First-order classical modal logic. Studia Logica $84(2), 171-210$.

Blackburn, P., M. de Rijke, and Y. Venema (2001). Modal Logic. Cambridge University Press.

Brauner, T. and S. Ghilardi (2007). First-order modal logic. In P. Blackburn, J. van Benthem, and F. Wolter (Eds.), Handbook of Modal Logic. Elsevier.

Calardo, E. and A. Rotolo (2014). Variants of multi-relational semantics for propositional non-normal modal logics. Journal of Applied Non-Classical Logics 24(4), 293-320.

Chellas, B. (1980). Modal logic: an introduction. Cambridge University Press.

Corsi, G. (2002). A unified completeness theorem for quantified modal logics. Journal of Symbolic Logic 67(4), 1483-1510.

Elgesem, D. (1997). The modal logic of agency. Nordic Journal of Philosophical Logic 2, $1-46$.

Fagin, R., J. Y. Halpern, Y. Moses, and M. Y. Vardi (1995). Reasoning about Knowledge. MIT Press.

Fitting, M. and R. L. Mendelsohn (1998). First Order Modal Logic. London: Kluwer Academic Publishers.

Gabbay, D. (1976). Investigations in modal and tense logics with applications to problems in Philosophy and Linguistics. Reidel.

Gabbay, D., V. Shehtman, and D. Skvortsov (2009). Quantification in nonclassical logic. Number v. 1 in Studies in logic and the foundations of mathematics. Elsevier.

Garson, J. (2001). Quantification in modal logic. In D. Gabbay and F. Guenthner (Eds.), Handbook of Philosophical Logic, 2nd edition. Kluwer.

Garson, J. (2005, October). Unifying Quantified Modal Logic. Journal of Philosophical Logic 34(5), 621-649.

Gasquet, O. and A. Herzig (1996). From classical to normal modal logic. In H. Wansing (Ed.), Proof Theory of Modal Logic, pp. 293-311. Dordrecht: Kluwer.

Goble, L. (2001). Multiplex semantics for deontic logic. Nordic Journal of Philosophical Logic 5(2), 113-134.

Goble, L. (2003). Preference semantics for deontic logic part I-Simple models. Logique et Analyse 46, 383-418.

Goble, L. (2004a). Preference semantics for deontic logic — Part II: Multiplex models. Logique et Analyse 47, 113-134.

Goble, L. (2004b). A proposal for dealing with deontic dilemmas. In A. Lomuscio and D. Nute (Eds.), Deontic Logic, Volume 3065 of Lecture Notes in Computer Science, pp. 74-113. Springer Berlin - Heidelberg.

Goble, L. (2005). A logic for deontic dilemmas. J. Appl. Log. 3(3-4), 461-483. 
Goldblatt, R. (1992). Logics of Time and Computation (2. ed.). Number 7 in CSLI Lecture Notes. Stanford, CA: Center for the Study of Language and Information.

Goldblatt, R. (2011). Quantifiers, Propositions and Identity: Admissible Semantics for Quantified Modal and Substructural Logics. Cambridge University Press.

Governatori, G. and A. Rotolo (2005). On the axiomatization of Elgesem's logic of agency and ability. Journal of Philosophical Logic 34(4), 403-431.

Hansen, H. H. (2003). Monotonic Modal Logics. Master's thesis, ILLC, Universiteit van Amsterdam, Amsterdam.

Jennings, R. E. and P. K. Schotch (1981a). Non-Kripkean Deontic Logic. In R. Hilpinen (Ed.), New studies in deontic logic: norms, actions, and the foundations of ethics, pp. 149-162. Dordrecht, Holland: D. Reidel Publishing Company.

Jennings, R. E. and P. K. Schotch (1981b). Some remarks on (weakly) weak modal logics. Notre Dame J. Formal Logic 22(4), 309-314.

Jones, A. and J. Carmo (2002). Deontic logic and contrary-to-duties. In D. Gabbay and F. Guenthner (Eds.), Handbook of Philosophical Logic (2nd ed.). Dordrecht ; Boston: Kluwer Academic Publishers.

Kracht, M. and F. Wolter (1999). Normal monomodal logics can simulate all others. J. Symb. Log. 64(1), 99-138.

Kripke, S. (1963). Semantical considerations on modal logic. Acta Philosophica Fennica 16, 83-94.

Kripke, S. (1980). Naming and Necessity. Blackwell Publishers.

Montague, R. (1970). Universal grammar. Theoria 36(3), 373-98.

Parikh, R. (1985). The logic of games and its applications. Annals of Discrete Mathematics 24, 111-140.

Pauly, M. (2002). A modal logic for coalitional power in games. Journal of Logic and Computation 12, 149-166.

Scott, D. (1970). Advice in modal logic. In R. Hilpinen (Ed.), Philosophical Problems in Logic, pp. 143-173. Dordrecht: Reidel.

Segerberg, K. (1971). An Essay in Classical Modal Logic (Uppsala Universitet ed.), Volume 13. Uppsala: Filosofiska Studier.

Segerberg, K. (1992). Getting started: Beginnings in the logic of action. Studia Logica $51(3 / 4), 347-378$.

Stolpe, A. (2003). QMML: Quantified minimal modal logic and its applications. Logic Journal of the IGPL 11(5), 557-575.

Waagbø, G. (1992). Quantified modal logic with neighborhood semantics. Zeitschrift für Mathematische Logik und Grundlagen der Mathematik 38, 491-499. 